\title{
On the symmetry of the universe
}

\section{Engel Roza}

Philips Research Labs, Eindhoven, The Netherlands (retired)

Email: engel.roza@onsbrabantnet.nl

\section{Summary}

It is shown that the Lambda component in the cosmological Lambda-CDM model can be conceived as vacuum energy, consisting of gravitational particles subject to Heisenberg's energy-time uncertainty. These particles can be modelled as elementary polarisable Dirac-type dipoles ("darks") in a fluidal space at thermodynamic equilibrium, with spins that are subject to the Bekenstein-Hawking entropy. Around the baryonic kernels, uniformly distributed in the universe, the spins are polarized, thereby invoking an increase of the effective gravitational strength of the kernels. It explains the dark matter effect to the extent that the numerical value of Milgrom's acceleration constant can be assessed by theory. Non-polarized vacuum particles beyond the baryonic kernels compose the dark energy. The result is a quantum mechanical interpretation of gravity in terms of quantitatively established shares in baryonic matter, dark matter and dark energy, which correspond with the values of the LambdaCDM model..

Keywords: Milgrom's acceleration constant; Bekenstein-Hawking entropy; gravitational dipole; dark matter.

\section{Introduction}

Although symmetry and entropy are closely related concepts, they are not the same. Whereas entropy can be unambiguously defined in terms of the Boltzmann conjecture as the logarithm of the number of indistinguishable microstates in a physical system, symmetry has a twofold ambiguity. On the one hand, one might say that a physical system consisting of elementary particles in free chaotic motion shows a maximum amount of (high) symmetry in its state of thermal equilibrium, on the other hand one might say that a nicely ordered physical system, in which elementary particles are structurally bound, has a high degree of symmetry. Whereas in the first case the high symmetry coincides with a high degree of entropy, the high symmetry in the second case is obtained from a loss in entropy [1]. This loss is usually denoted as a spontaneous symmetry break.

This correspondence and difference shows up as well in the search toward the relationship between quantum mechanics and gravity. This subject has given rise to a discipline in itself, dubbed as quantum gravity. Within this discipline, new concepts are being developed, such as for instance the string theory. Those new concepts are often denoted as new physics. It is believed that such new concepts will explain certain phenomena in cosmology, where dark matter and dark energy are still regarded as a mystery. An interesting approach is building new physics emergently from basic principles, such as for instance from the entropy concept as known from thermodynamics. About some ten years ago, proposals in this direction have been put forward by Verlinde [2,3], inspired by views of Jacobson [4] back in 1995. These proposals have resulted into a particular approach to gravity, known as entropic gravity. Verlinde's theory aims to connect entropic gravity with string theoretic principles and information theory. The drawback of this approach for a scientist with an interest in the subject is the need to cope with the highly sophisticated mathematical mask that covers the theory. It is my aim to reverse the argumentation. Rather than showing how Einstein's theory of gravity emerges from basic principles, I want to show that the basic principles emerge from Einstein's theory, even to the extent of the role of informatics and thermodynamic entropy. It is my belief that this will result in easier understanding of the very same principles and in quantitative 
results that give a better fit to observational evidence. One of the results, next to understanding the role of informatics in gravity, is a very clear explanation of the dark matter phenomenon in terms of a symmetry break, culminating in a quantitative calculation of Milgrom's acceleration constant [5] from basic quantum mechanical principles, developed from conceiving the vacuum energy term in Einstein's equation as the Heisenberg fluctuation on the irrelevant thermal dynamical equilibrium state of spatial particles in a virtual fluid. In the present canonic cosmology model, known as the Lambda-C(old)D(ark)M(atter) model $(\Lambda \mathrm{CDM}$ ) as defined by the Planck Cooperation Group [6], dark matter is one of the three components of gravitational matter. The second one is the ordinary physical matter, observable as solar systems, galaxies and gas clouds, known as baryonic matter, and the third one is the even more mysterious dark energy, which is responsible for the accelerated expansion of the visible universe. It is another aim in this article to develop a physically understandable model of the vacuum energy that allows a theoretical assessment of the ratios between the three components.

Accepting the concept of elementary particles (within the scope of this article indicated as "darks"'") in a fluidal energetic vacuum, raises an interest into their entropic behavior. The first issue that I want to discuss is the question whether it is possible to establish a figure for the particle's volume density such that Milgrom's acceleration constant can be related with the entropy measure of the visible universe. This question will be addressed in paragraphs 2-4. This includes a development of a model for the elementary energetic constituent (dark). Subsequently, in paragraphs 5-6, the entropicly established particle's volume density will be validated by theory. This includes an uncommon view on the role of Einstein's $\Lambda$ in his Field Equation, which results into a modification of Newton's gravity law. In paragraph 7, the theory is extended from solar systems and galaxies to the universe with a uniform distribution of galaxies. This precedes a discussion paragraph and a conclusive reflection on the symmetry of the universe. An appendix is added showing the details how Einstein's $\Lambda$ influences the Newtonian gravity.

\section{Gravitational entropy}

Let me start by sketching the relationship between Milgrom's acceleration constant of dark matter with the well known entropy expression for black holes as eventually established by Hawking as an improvement of Bekenstein's original formulation. In this respect it is not different from like it is in Verlinde's artcle [3]. The theory, the interpretation and the results, though, are quite different. The Bekenstein-Hawking expression is one of five ingredients for calculation. It reads as $[7,8]$,

$S_{H}=k_{B} \frac{c^{3}}{4 G \hbar} A$

where $c$ is the vacuum light velocity, $G$ the gravitational constant, $\hbar$ Planck's (reduced) constant, $A$ the black hole's peripheral area and $k_{B}$ is Boltzmann's constant. The peripheral area of a spherical black hole is determined by its Schwarzschild radius as,

$A=4 \pi R_{S}^{2} ; R_{S}=\frac{2 M G}{c^{2}}$,

In which $M$ is the baryonic mass of the black hole. Boltzmann's constant shows up as a consequence of the thermodynamic definition of entropy. In that definition $S_{H}$ is not dimensionless, because of the thermodynamic interpretation of entropy as a measure for the unrest of molecules due to 
temperature, which relates the increase $\Delta S$ of entropy with an increase of the molecular energy $\Delta E$ due to temperature $T$, such as expressed by the thermodynamic definition,

$\Delta E=T \Delta S$

Boltzmann's famous conjecture connects entropy with information, by stating

$S_{B}=k_{B} \log (\#$ microstates).

This conjecture expresses the expectation that entropy can be expressed in terms of the total number of states that can be assumed by an assembly of molecules. Boltzmann's constant shows up to correct for dimensionality. I would like to emphasize here that (3) and (4) are different definitions for entropy $S$, and are not necessarily identical. Knowing that (1) has been derived from (3) and accepting Boltzmann's conjecture, we would have,

$\frac{c^{3}}{4 G \hbar} A=\log (\#$ microstates $)$

Both sides of this expression are dimensionless. Omitting Boltzmann's constant makes entropy a dimensionless measure of information, which, of course, is very appealing. At this point, I wish to elaborate on a subtlety, which has been shown by Verlinde. According to Boltzmann's conjecture, an elementary step $\Delta S$ in entropy would imply $\Delta S=k_{B}$. Verlinde has proven, however, that an elementary step in entropy from the Hawking-Bekenstein entropy implies $\Delta S=2 \pi k_{B}$. If not, the Hawking-Bekenstein's formula would violate Newton's gravity law [2]. Because Boltzmann's expression is a conjecture without proof, the problem can be settled by modifying the dimensionless expression of entropy (5) into,

$S=\frac{1}{2 \pi} \frac{c^{3}}{4 G \hbar} A=\log ($ \#microstates $)$

It is instructive comparing this result with a view on the Bekenstein-Hawking entropy as suggested by Susskind (for explanation purpose) in one of his lectures [9]. Susskind has proposed to consider the black hole as a body that captures or releases elementary discrete packages of energy $\Delta M=h f=\hbar \omega$, building its total mass $M$ as a sum of $N$ elementary amounts $\Delta M$. Each of these elementary packages add an elementary step on entropy. To this end, the Compton wavelength of these elements must equate the peripheral circle $2 \pi R_{S}$ of the black hole, such that

$\Delta M=h f=h \frac{1}{T}=\frac{h c}{c T}=\frac{h c}{2 \pi R_{S}}=\frac{\hbar c}{R_{S}}$.

In Susskind's explanation model, the entropy is equated with the ratio,

$$
S=N=\frac{M}{\Delta M}=\frac{R_{S}}{\hbar c} M=\frac{R_{S}}{\hbar c}\left(\frac{2 M G}{c^{2}}\right) \frac{c^{2}}{2 G}=\frac{c^{3}}{2 \hbar G} R_{S}^{2}=\frac{c^{3}}{2 \hbar G} \frac{A .}{4 \pi}=\frac{1}{2 \pi} \frac{c^{3}}{4 \hbar G} A .
$$

This result is in agreement with (6). 
The second ingredient is the well known observation that the event horizon $c t_{H}$ of the visible universe equals Schwarzschild radius of the critical mass enclosed within that horizon $\left(t_{H}\right.$ is the Hubble time scale ), [10]. The baryonic share in this critical mass amounts to $\Omega_{B}$. Hence, from (6), the Bekenstein-Hawking entropy within the event horizon of the "flat" universe can be established as

$S=\Omega_{B} \frac{c^{3}}{8 \pi G \hbar} 4 \pi\left(c t_{H}\right)^{2}$

Note: In terms of the Lamda-CDM nomenclature, the baryonic share is expressed as $\Omega_{B}$ in the relationship

$1=\Omega_{m}+\Omega_{\Lambda}=\left(\Omega_{B}+\Omega_{D}\right)+\Omega_{\Lambda}$,

where $\Omega_{m}, \Omega_{\Lambda}, \Omega_{B}, \Omega_{D}$, respectively are the relative matter density, the relative dark energy matter density, the relative baryonic matter density and the relative dark matter density [6]. Where the matter distribution between the matter density $\Omega_{m}(=0.259)$ and dark energy density $\Omega_{\Lambda}(=$ 0.741 ) is largely understood as a consequence from the Friedmann equations [19] that evolve from Einstein's Field Equation under the Friedmann-Lemairtre-Robertson-Walker (FLRW) metric [20], the distribution between the baryonic matter density $\Omega_{B}(=0.0486)$ and dark matter density $\Omega_{D}(=$ 0.210 ) is empirically established from observation. The quoted values are those as established by the Planck Collaboration [6].

\section{Constituents of the vacuum energy ("darks")}

The third ingredient is crucial novel step. It is a consideration on the vacuum energy of the universe. Let us suppose that the origin of gravitational energy is due to the Heisenberg uncertainty of elementary spatial particles. Let us suppose, just by hypothesis, that their position $d$ in their center of mass frame can be explained as the result of a motion with ultra-relativistic speed near vacuum light velocity $c$, such that

$d=c \Delta t$

Applying Heisenberg's relationship $\Delta E \Delta t=\hbar / 2,[11]$, on (10), we get

$$
d=c \Delta t=c \frac{\hbar}{2} \frac{1}{\Delta E} \rightarrow d=c \frac{\hbar}{2} \frac{1}{m c^{2}} \rightarrow p=m d=\frac{\hbar}{2 c},
$$

in which $p$ has the dimensions of a (mass) dipole moment expressed in terms of Planck's reduced constant $\hbar$ and the vacuum light velocity $c$. The virtual mass $m$ should not be confused with the particle's rest mass $m_{0}$. This implies, so far, that elementary spatial particles can be modelled by gravitational dipoles with a dipole moment $p$ as described by (12). Note that, where other authors $[13,14]$ describe gravitational dipoles as structures with positive and negative mass ingredients, the dipole described here is a virtual vibrating particle. To some readers it may 
seem that I am introducing here a new kind of matter. This is not true. The vibrating particle is part of the vacuum energy, modelled as an ideal fluid in thermodynamic equilibrium that emerges from Einstein's Lambda in the solution of his Field Equation of the vacuum [15,16,17]. The equilibrium state of the fluid itself is irrelevant. Hence the gravitational molecules show up as a vibration of the vacuum. This is different from novel matter of baryonic nature.

It is true, though, that it may seem that the existence of particles with a dipole moment defined by (12) cannot be related with known elementary fermions. However, it has been proven quite recently that, next to the electron-type and the Majorana type, Dirac's theory of electrons predicts a third type with the unique property of showing a real dipole moment

$$
\boldsymbol{p}=\bar{\sigma} h / c,
$$

in which $\bar{\sigma}$ is the Pauli vector with eigen value $|\bar{\sigma}|=1 / 2$ and which, unlike the two other ones, is polarisable in a scalar potential field [18]. Originating from the Heisenberg uncertainty, this polarisable dipole moment is a pure quantum mechanical phenomenon. Its dipole mass is unrelated with the rest mass of the particle. The rest mass may have any value, down to an extremely tiny quantity, while leaving the dipole moment unaffected. This property fits well to the gravitational "dark" as just described.

\section{The gravitattional dipole density (1)}

The fourth ingredient is the dipole density $P_{g}$. Its assessment is far from trivial. Eventually, it will appear being

$$
P_{g}=\frac{a_{0}}{20 \pi G}
$$

in which $a_{0}$ is just a constant with the dimensionality of acceleration. Later in this article, it will be identified as Milgrom's acceleration constant of dark matter [5]. This relationship will be developed in the next two paragraphs. Taking this result for the moment as granted, we may from (12) and (14) calculate the amount $N_{g}$ of gravitational dipoles in the spatial volume $V$ enclosed by the event horizon of the universe, as

$$
N_{g}=\frac{P_{g} V}{p} ; V=\frac{4}{3} \pi\left(c t_{H}\right)^{3} .
$$

Hence, the amount of dipoles in a spatial volume $V$ is established from (14-15) as,

$$
N_{B}=\frac{P_{g}}{p} V=\frac{a_{0}}{20 \pi G} \frac{2 c}{\hbar}\left(\frac{4}{3} \pi c^{3} t_{H}^{3}\right) \text {. }
$$

Their spin states (= direction of the dipole moment vector) carry the amount of information contained in the universe. Because the quantum states of the spin can assume two values only, the 
number $N_{B}$ are bits that represent the baryonic information content of a space with volume $V$. Hence, $S$ as defined by $(1,2,6)$ and $N_{B}$ as defined by $(16)$, both express the entropy of the volume enclosed within the event horizon of the universe. Hence, equating $S$ and $N_{B}$ yields the instrument for the calculation of the acceleration constant $a_{0}$. Doing so, we find from (16) and (9),

$a_{0}=\frac{15}{4} \Omega_{B} \frac{c}{t_{H}}$.

From (17), we find, with $\Omega_{B}=0.0486, c=3 \times 10^{8} \mathrm{~m} / \mathrm{s}$ and the Hubble time scale $t_{H}=13.8$ Gyear, $a_{0} \approx 1.25 \times 10^{-10} \mathrm{~m} / \mathrm{s}^{2}$, which corresponds extremely well with Milgrom's empirical acceleration constant indeed $[5,21]$.

It has to be emphasized that this result relies upon (a) the modelling of the universe as a virtual black hole, (b) filled with energetic particles (darks) uniformly distributed with a particular density, (c) in a particular mix of baryonic matter ones, dark matter ones and dark energy ones. Where the virtual black hole model of the universe and the energy density distribution (10) is common knowledge, the particular particle density as shown by (15) needs proof. This will be the subject of the next paragraphs..

\section{Einstein's Lambda and the Cosmological Constant}

Let us first consider the problem how to prove relationship (14). Because Einstein's Lambda in his Field Equation embodies the cosmological background energy, a close inspection of this famous equation is required. This Field Equation reads as,

$G_{\mu v}+\Lambda g_{\mu v}=\frac{8 \pi G}{c^{4}} T_{\mu \nu} ; G_{\mu \nu}=R_{\mu \nu}-\frac{1}{2} R g_{\mu \nu}$

in which $T_{\mu \nu}$ is the stress-energy function, which describes the energy and the momenta of the source(s) and where $R_{\mu \nu}$ and $R$ are respectively the so-called Ricci tensor and the Ricci scalar. These can be calculated if the metric tensor components $g_{\mu \nu}$ are known [22,23,24]. The $\Lambda$ term is missing in Einstein's paper of 1916, in spite of his awareness that he equated an integration constant as zero (see footnote on p.804 in ref. [22]). Later, in 1917, Einstein added this quantity as a covariant integration constant for allowing vacuum solutions of his Field Equation [15,25]. It is usually presently taken for granted that this Lambda is a Cosmological Constant that can be regarded as a constant of nature. In fact, however, it is just a constant in the sense that its value does not depend on time-space coordinates. Hence, it may have different values depending on the scope of a cosmological system under consideration, because it may depend on attributes, like mass content for instance. It may have different values at the level of solar systems, galaxies and the universe. Only at the latter level, it is justified to identify the $\Lambda$ as the Cosmological Constant indeed. At that 
level, by the way, the cosmological system is in a state of maximum symmetry and maximum entropy. Generally, however, $\Lambda$ is just a constant in Einstein's Field Equation.

In the case that a particle under consideration is subject to a central force only, the timespace condition shows a spherical symmetric isotropy. This allows to read the metric elements $g_{i j}$ from a simple line element that can be written as

$\mathrm{d} s^{2}=g_{t t}(r, t) \mathrm{d} q_{0}^{2}+g_{r r}(r, t) \mathrm{d} r^{2}+r^{2} \sin ^{2} \vartheta \mathrm{d} \varphi^{2}+r^{2} \mathrm{~d} \vartheta^{2}$

where $q_{0}=\mathrm{i} c t$ and $\mathrm{i}=\sqrt{-1}$.

It means that the number of metric elements $g_{i j}$ reduce to a few, and that only two of them are time and radial dependent.

Note: The author of this article has a preference for the "Hawking metric" $(+,+,+,+)$ for (ict, $x, y, z$ ), like, for instance also used by Perkins [26]. By handling time as an imaginary quantity instead of a real one, the ugly minus sign in the metric $(-,+,+,+)$ disappears owing to the obtained full symmetry between the temporal domain and the spatial one.

Before discussing the impact of $\Lambda$, it is instructive to summarize Schwarzschild's solution of Einstein's equation for a central pointlike source in empty space and $\Lambda=0$, in which the metric components appear being subject to the simple relationship

$$
g_{r r} g_{t t}=1
$$

Solving Einstein's equation under adoption of a massive source with pointlike distribution $T_{00}=M c^{2} \delta^{3}(r)$, results in a wave equation with the format [24],

$$
-\frac{\partial^{2} g_{t t}}{c^{2} \partial t^{2}}+\frac{1}{r} \frac{\partial^{2}\left(r g_{t t}\right)}{\partial r^{2}}=-\frac{8 \pi G M}{c^{2}} g_{r r} \delta^{3}(r) U(t),
$$

where $U(t)$ is Heaviside's step function.

Its stationary solution under the weak field limit

$$
g_{t t}(r, t)=1+h_{\varphi}(r, t), \text { in which }\left|h_{\varphi}(r, t)\right|<<1,
$$

is the well-known Newtonian potential,

$$
\Phi=-\frac{M G}{r}, \text { where } h_{\varphi}=\frac{2 \Phi}{c^{2}}
$$

The wave equation (21) reduces to, 
$-\frac{\partial^{2}}{c^{2} \partial t^{2}}(r \Phi)+\frac{\partial^{2}}{\partial r^{2}}(r \Phi)=-r \frac{8 \pi G M}{c^{2}} \delta^{3}(r) U(t)$

With inclusion of the constant $\Lambda$, the wave equation is modified to (see Appendix),

$$
-\frac{\partial^{2} g_{t t}}{c^{2} \partial t^{2}}+\frac{1}{r} \frac{\partial^{2}\left(r g_{t t}\right)}{\partial r^{2}}+2 \Lambda=-g_{r r} \frac{8 \pi G T_{t t}}{c^{2}} \delta^{3}(r) U(t) .
$$

If $T_{t t}$ were a pointlike source $T_{t t}=M c^{2} \delta^{3}(r) U(t)$, where $U(t)$ is Heaviside's step function, the static solution of this equation would be provided by the Schwarzschild-de Sitter metric, also known as Kottler metric, $[27,28]$, given by

$g_{t t}(r)=\frac{1}{g_{r r}(r)}=1-\frac{R_{S}}{r}-\frac{\Lambda}{3} r^{2}$, with $\quad R_{S}=\frac{2 M G}{c^{2}}$

The viability of (26) into (25) readily follows by insertion into (25) and subsequent evaluation. Obviously, we meet a problem here, because we cannot separate a weak field $\Phi$ (= gravitational potential) from the metric, because a priori we cannot identify an $r-$ domain that justifies the adoption of the constraint (22). However, given the fact that a viable wave function can be obtained for $\Lambda=0$, one might expect that it must be possible to obtain a valid wave equation for a weak field $\Phi$ showing a gradual move from $\Lambda=0$ to $\Lambda \neq 0$. The way out from the problem is the consideration that (25) must be valid both for vacuum with a massive source as well as for vacuum without a source. Taking the view that the vacuum is something else but empty space, allows as I wish to show, a meaningful wave equation. This starts by the observation that the Kottler metric describes the space-time curving of a spherical empty space under influence of a single central pointlike energy source. But in our view there is not such a thing as empty space. Instead there is a vacuum composed by energetic vacuum particles.

Hence, where an empty space with $\Lambda=0$ corresponds with virtual sources $T_{\mu \mu}=0$, the vacuum with $\Lambda \neq 0$ is a fluidal space with virtual sources $T_{\mu \mu}=-p \Lambda$, with $g_{\mu \mu}=\left(1,1, r^{2} \sin ^{2} \vartheta, r^{2}\right)$, where $p=c^{4} / 8 \pi G[17,29,30]$. (Owing to the Hawking metric, $p$ is equal for all diagonal elements). This particular stress-energy tensor with equal diagonal elements corresponds with the one for a perfect fluid in thermodynamic equilibrium [31]. Inserting a massive source in this fluid will curve the vacuum to $g_{\mu \mu}=\left(g_{t t}, g_{r r}, r^{2} \sin ^{2} \vartheta, r^{2}\right)$. Hence, inclusion of the Cosmological Constant $\Lambda$ implies that, under absence of massive sources, Einstein's equation can be satisfied if empty space is given up and is replaced by a space that behaves as a molecular fluidum in thermodynamic equilibrium. If, under bias of a uniformly distributed background energy, a massive pointlike source is inserted into this fluidum, deriving a meaningful wave equation is possible, although not trivial. As shown in the Appendix, the difficulty is mainly caused by the loss in symmetry between $g_{t t}$ and $g_{r r}$. Whereas Schwarzschild's relationship (20), implies a weak field limit, 


$$
h_{t \varphi}=-h_{r \varphi}
$$

the loss of the relationship implies,

$\left|h_{t \varphi}\right| \neq\left|h_{r \varphi}\right|$.

It also means that it is no longer clear how to relate a Newtonian potential $\Phi$ with the metric components, such as shown by (23). To make things even worse, the consequences in all four metric components $g_{t t}, g_{r r}, g_{\vartheta \vartheta}, g_{\varphi \varphi}$ have to be considered. Nevertheless, it will appear (derived in the Appendix) that the low value of Einstein's $\Lambda$ still allows a linearization of the equation set within a conditioned spatial range. This will give rise to a wave equation with the format,

$-\frac{\partial^{2}}{c^{2} \partial t^{2}}(r \Phi)+\frac{\partial^{2}}{\partial r^{2}}(r \Phi)+\lambda^{2}(r \Phi)=-r \frac{8 \pi G M}{c^{2}} \delta^{3}(r) U(t)$

In which $\lambda^{2}=2 \Lambda$ and in which $\frac{2 \Phi}{c^{2}}=h_{t \varphi}\left(\neq-h_{r \varphi}\right)$.

While this wave equation in the weak field limit is compatible with the behavior of $g_{t t}$, the behavior of $g_{r r}$, being different from $g_{t t}$, might spoil the weak field limit condition. As shown in the Appendix, this is not the case as long as $\lambda r<\approx 6$. This sets an upper limit to the validity of (28). Another concern is the possible violation of the metric (19) due to the behavior of the metric components $g_{\vartheta \vartheta}$ and $g_{\varphi \varphi}$ under $\Lambda \neq 0$. This sets a lower limit to the validity of (28). This condition, being derived in the Appendix as well, will be discussed later in this article.

The main message so far is, that the curving of space-time as a consequence of inserting a massive pointlike source in empty space, like assumed under the Schwarzschild-de Sitter condition and the Kottler condition, is different from the curving of space-time in the case of inserting a massive source in a fluidal vacuum. The Appendix serves to show the details of the derivation. The static format of the wave equation (28) is a potential field set up by a pointlike source with a format that shows up as a modification of Poisson's equation, such that

$$
\frac{\partial^{2}}{\partial r^{2}}(r \Phi)+\lambda^{2}(r \Phi)=-r \frac{8 \pi G M}{c^{2}} \delta^{3}(r)
$$

This result is different from the common perception on the influence of Einstein's $\Lambda$ in Newtonian gravity. Like discussed in review articles on its history, such as for instance by Norton [32] and by Harvey and Schucking [33], the resulting Laplace equation should match with (25) such that,

$$
\frac{1}{r} \frac{\partial^{2}}{\partial r^{2}}\left(r \Phi^{\prime}\right)+\lambda^{2}=0
$$


This latter one is compatible with the de Sitter-Schwarzschild metric (26).

The difference between (29) and the commonly accepted view (30), touches the crux of this article. Where (30) traces back to the solution of Einstein's equation given by de SitterSchwarzschild metric, solution (29) traces back to the fluidal space solution. It has to be emphasized here that comparing (29) and (30) is comparing apples with pears, because of the semantic difference between $\Phi^{\prime}$ and $\Phi$. Where $\Phi^{\prime}$ applies to $g_{t t}, \Phi$ applies to $h_{\varphi}$, which is a small deviation on top of $g_{t t}$, as defined by (22). What eq.(29) actually expresses, is a tiny change in the space-time curving on top of the one that is caused by an energetic source. If the source is missing, there would be no curving, neither a change (zero $\left.h_{\varphi}\right)$. In that case, (29) simply reduces to the identity $0 \equiv 0$. Where eq. (30) is made up at the cosmological level of the whole universe (modelled as a de Sitter space), (29) is made up for a spherical system with a central mass, such as applies to solar systems and galaxies. As we shall demonstrate later in this article, this has an impact on the interpretation of the Cosmological Constant.

As noted before and well known of course, Poisson's equation and its modification, is the static state of a wave equation. From the perspective of classic field theory, a wave equation, can be conceived as the result of an equation of motion derived under application of the action principle from a Lagrangian density L of a scalar field with the generic format

$$
\mathrm{L}=-\frac{1}{2} \partial_{\mu} \Phi \partial^{\mu} \Phi+U(\Phi)+\rho \Phi
$$

where $U(\Phi)$ is the potential energy of the field and where $\rho \Phi$ is the source term. Comparing various fields of energy, we have,

$$
\begin{array}{ll}
U(\Phi)=0 & \text { for electromagnetism. } \\
U(\Phi)=-\lambda^{2} \Phi^{2} / 2 & \text { for this case, } \\
U(\Phi)=\lambda^{2} \Phi^{2} / 2 & \text { for the nuclear forces [34] }
\end{array}
$$

The non-trivial solutions of (31) in homogeneous format, for the first case and the third case, are respectively,

$\Phi=\frac{\Phi_{0}}{\lambda r}$ and $\Phi=\Phi_{0} \frac{\exp (-\lambda r)}{\lambda r}$

The first case applies to electromagnetism (for $\Phi_{0}=Q \lambda / 4 \pi \varepsilon_{0}$ ) and to Newtonian gravity (for $\Phi_{0}=-M G \lambda$ ). The third case applies to Proca's generalization of the Maxwellian field [35]. The latter one reduces to the first case if $\lambda \rightarrow 0$, while keeping $\Phi_{0} / \lambda$ constant. Generically, it represents a field with a format that corresponds with the potential as in the case of a shielded electric field (Debije [36]), as well as with Yukawa's proposal [34], to explain the short range of the nuclear force. 
Let us, after this side-step, proceed on (29). It can be readily be verified that this equation can be satisfied by,

$$
\Phi=\Phi_{0} \frac{\cos \lambda r+\sin \lambda r}{\lambda r}
$$

Note that the goniometric shape of this solution is a consequence of the plus sign in front of $\lambda^{2}$. It has to emphasized once more that this expression holds under the classical weak field constraint and the presence of a central source of energy that evokes this field as the tiny variation in the generic spherical metric. The shape (34) will reveal some interesting features. In accordance with the concepts of classical field theory, the field strength can be established as the spatial derivative of the potential $\Phi$. Identifying $\Phi_{0} / \lambda$ as $-M G$ and $\lambda$ as a range parameter, we may identify this field strength as a cosmological gravitational acceleration $g$. Let us compare this acceleration with the Newtonian one $g_{N}$.

Hence, from

$$
\begin{aligned}
& \left.g=\frac{d \Phi}{d r}=\frac{\Phi_{0}}{\lambda r^{2}}\{(1-\lambda r) \cos \lambda r+(1+\lambda r) \sin \lambda r)\right\} ; \Phi_{0}=-M G \lambda \rightarrow \\
& \left.g=g_{N}\{(1-\lambda r) \cos \lambda r+(1+\lambda r) \sin \lambda r)\right\} ; g_{N}=\frac{M G}{r^{2}} .
\end{aligned}
$$

Not surprisingly, the gravitational acceleration is affected by the Einstein's $\Lambda=\lambda^{2} / 2$. If $\Lambda=0$, the gravitational acceleration equals the Newtonian one $g_{N}$. Under a positive value of the Cosmological Constant, the gravitational acceleration has a different spatial behavior. This is illustrated in figure 1 , which shows the ratio $g / g_{N}$ as a function of the normalized spatial quantity $\lambda r$. Up to the value $\lambda r=3 \pi / 4, g / g_{N}$ rises monotonously up to the value $g / g_{N}=3.33$. This figure shows that, for relative small values of $r$, the cosmological acceleration behaves similarly as the Newtonian one. Its relative strength over the Newtonian one increases significantly for large values of $r$, although it drops below the Newtonian one at $\lambda r>3.45$. Up to slightly below $\lambda r=3 \pi / 4$, this is, as will be shown, a similar behavior as heuristically implemented in MOND. The effective range is determined by the parameter $\lambda$. It might therefore well be that the cosmological gravity force manifests itself only at cosmological scale. Let us consider its consequence.

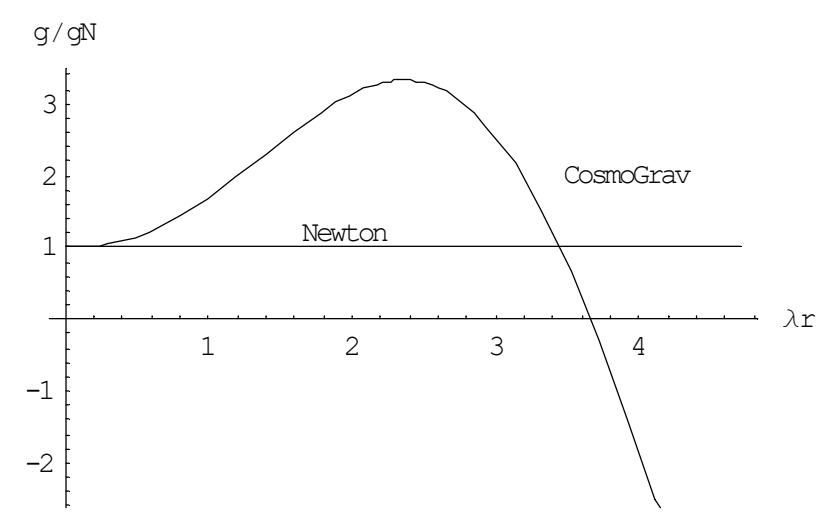


Figure 1: The cosmological gravity force compared with the Newtionian force

Newtonian laws prescribe that the transverse velocity $v_{\varphi}(r)$ of a cosmic object revolving in a circular orbit with radius $r$ in a gravity field is determined by

$$
v_{\varphi}^{2}(r)=\frac{M(r) G}{r},
$$

in which $M(r)$ is the amount of enclosed mass. This relationship is often denoted as Kepler's third law. Curiously, like first announced by Vera Rubin [37] in 1975, the velocity curve of cosmic objects in a galaxy, such as, for instance, the Milky Way, appears being almost flat. It is tempting to believe that this can be due to a particular spectral distribution of the spectral density to compose $M(r)$. This, however, cannot be true, because $M(r)$ builds up to a constant value of the overall mass. And Kepler's law states in fact that a flat mass curve $M(r)$ is not compatible with a flat velocity curve. Figure 2 illustrates the problem. It is one of the two: either the gravitational acceleration is, at cosmological distances, larger than the Newtonian one, or dark matter, affecting the mass distribution is responsible. Cosmological gravity as expressed by (35) may give the clue. Its effective range is determined by the parameter $\lambda$. It might therefore well be that the cosmological gravity force manifests itself only at cosmological scale. Figure 3 shows that under influence of this force, the rotation curves in the galaxy are subject to a boost.

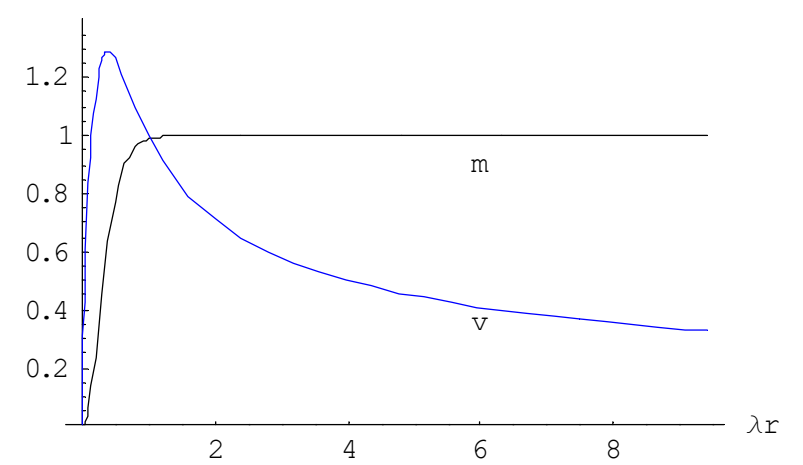

Figure 2. Incompatibility of a flat enclosed mass curve with a flat rotation curve. Note: for illustration purpose a particular distribution is adopted for the enclosed mass. The same distribution is maintained for all subsequent illustrations.

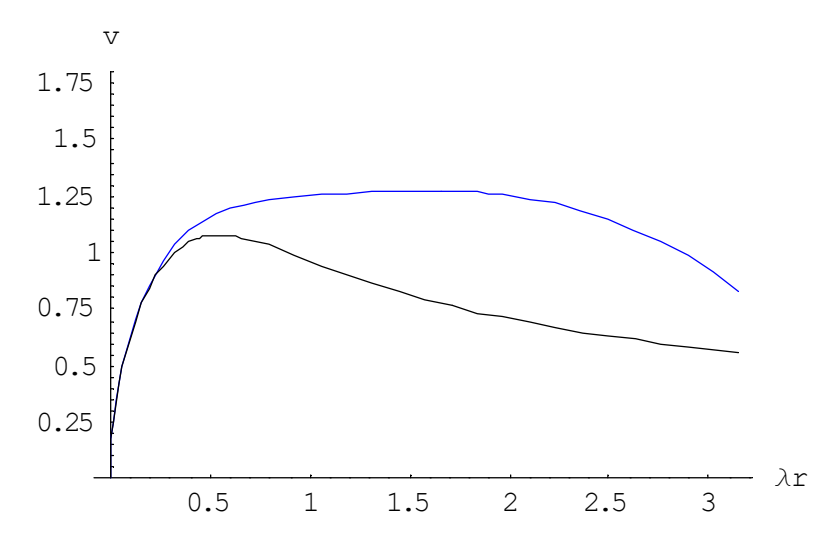

Figure 3: boost of the rotation curve under influence of cosmological gravity. 
This cosmological gravity shows another intriguing phenomenon. Like shown in figures 1 and 4 , at the very far cosmological distance, the attraction of gravity is inversed into repulsion $[38,39,40]$. However, rather than the "naked repulsion" (a word used by Harvey and Schucking [33]), like manifest in the de Sitter-Schwarzschild approach, the repulsion shows up at the very far end of the spatial range. Nevertheless, it prevents the clustering of the fluidal space, thereby eliminating the major argument against the fluidal space approach. Further exploration of this phenomenon is a subject outside the scope of this article. It has to be noted that the solution (35) is not unique. There are more solutions possible by modifying the magnitude of $\sin \lambda r$ over $\cos \lambda r$. I have simply chosen here for the symmetrical solution. Cosmological observations would be required to obtain more insight in this.

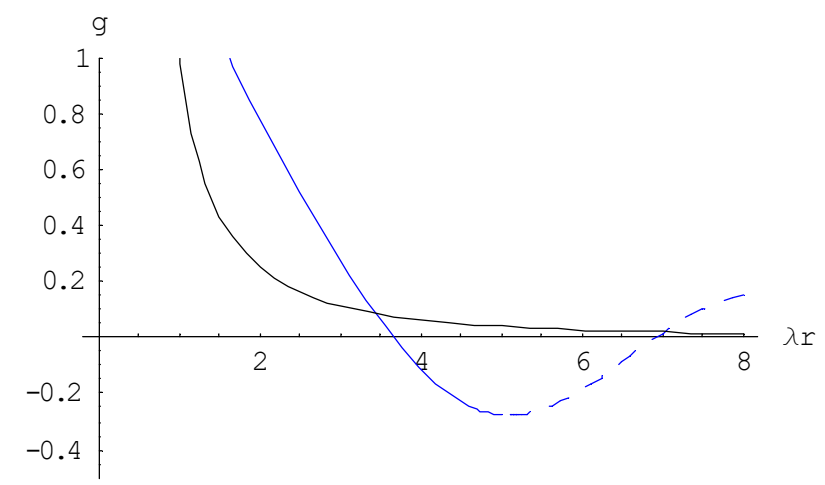

Figure 4: Inversion of the gravity force to antigravity at large cosmological distances. Black: Newtonian. Blue: Cosmological Gravity.

Whether this theoretically derived modification of the Newtonian gravity indeed explains the excessive orbital speeds of stars in a galaxy, such as formulated in Milgrom's empirical law in MOND, is dependent on the numerical value of Einstein's $\Lambda$. If Milgrom's empirical theory and the one developed in this paragraph are both true, it must be possible to relate Einstein's $\Lambda$ with Milgrom's acceleration constant $a_{0}$.

\section{Comparison with MOND}

MOND is a heuristic approach based on a modification of the gravitational acceleration $g$ such that

$$
g=\frac{g_{N}}{\mu(x)}, \text { with } x=g / a_{0}
$$

In which $\mu(x)$ is an interpolation function, $g_{N}\left(=M G / r^{2}\right)$ the Newtonian gravitational acceleration and in which $a_{0}$ is an empirical constant acceleration. The format of the interpolation function is not known, but the objectives of MOND are met by a simple function like $[5,21]$

$$
\mu(x)=\frac{x}{\sqrt{1+x^{2}}} .
$$


If $g / a_{0}<<1$, such as happens for large $r,(37)$ reduces to

$g=\sqrt{a_{0} g_{N}}$

Under this condition, the gravitational acceleration decreases as $r^{-1}$ instead of $r^{-2}$. As a result, the orbital velocity curves as a function of $r$ show up as flat curves.

Algebraic evaluation of (37) and (38) results into,

$$
\frac{g}{g_{N}}=\sqrt{\frac{1+\sqrt{1+4 k^{2}(\lambda r)^{4}}}{2}} \quad \text { with } k=\frac{a_{0}}{M G \lambda^{2}} \text {. }
$$

This expression allows a comparison with (35).

As illustrated in figure 5, a pretty good fit is obtained between (35) and (40) in the range up to $\lambda r=3 \pi / 4$ (where the theoretical curve starts decaying), if

$$
k=\frac{a_{0}}{M G \lambda^{2}}=2.5 \rightarrow a_{0}=2.5 M G \lambda^{2}=5 M G \Lambda
$$

Observations on various galaxies have shown that $a_{0}$ can be regarded as a galaxyindependent constant with a value about $a_{0} \approx 1.25 \times 10^{-10} \mathrm{~m} / \mathrm{s}^{2}$.

The implication of (41) is, that $a_{0}$ is a second gravitational constant next to $G$. The two constants determine the range $\lambda$ of the gravitational force in solar systems and galaxy systems as $\lambda^{2} \approx 2 a_{0} / 5 M G$, in which $M$ is the enclosed mass in those systems. Whereas this second gravitational quantity $a_{0}$ is an invariable constant, this is apparently not true for the Einsteinean parameter $\Lambda$.

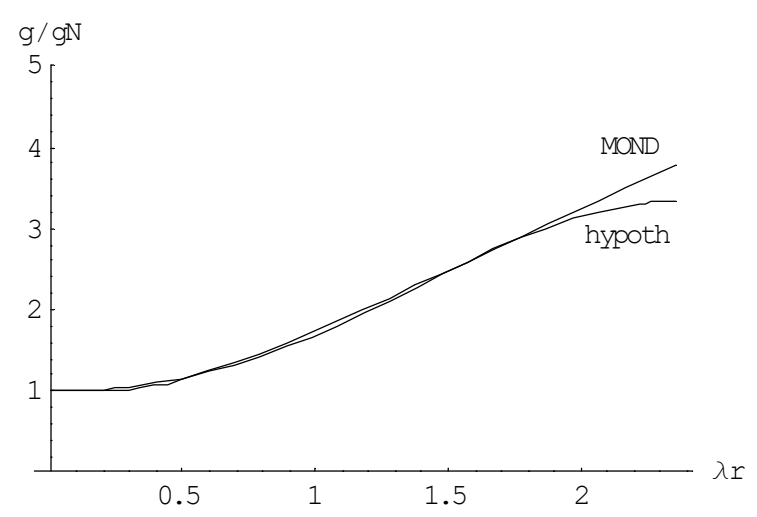

Figure 5: MOND's interpolation function compared with the theory as developed. 


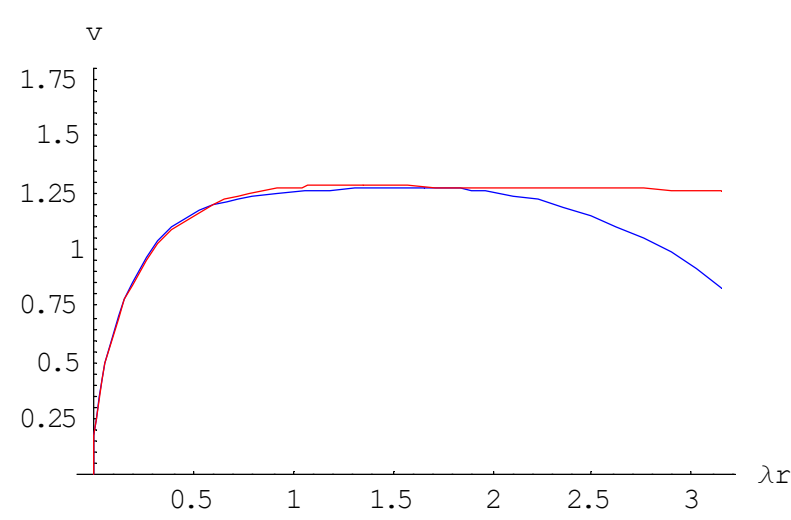

Figure 6: Comparison of orbital velocities for stars in galaxies for MOND (upper curve) and for the theory as developed (lower curve).

This result shows that Milgom's empirical and the theory as developed in this article are intimately related. Figure 6 shows the difference between curves for the orbital velocity of stars in galaxies according to MOND as compared to those as predicted by the theory as developed in this article. It has to be emphasized here that establishing the fit between the two curves by setting $k=2.5$ is only meant to incorporate Milgrom's acceleration constant $a_{0}$ as an unknown parameter into the theory. This implies that no limitation on whatsoever is imposed, nor that the generality of the analysis is affected. One may ask: "proves MOND the theory or does the theory proves MOND?". Maybe, the better question is: does the well accepted MOND validate the theory? To answer this question, an obvious difference between MOND and the developed theory has to be discussed. From figure 5 it is shown that beyond $r \lambda=3 \pi / 4$ the developed theory deviates from MOND and figure 4 shows that beyond $r \lambda=3.66$ the gravitational attraction changes into a repulsion. From this perspective, the latter phenomenon would even put a natural limit to the size of a galaxy. Let us consider these ranges for the Milky Way. Under the constraint (30), we have

$$
1 / \lambda=\sqrt{5 M G / 2 a_{0}} .
$$

The spatial coincidence range between MOND and the theory developed in this article is up to

$$
R_{M}=\frac{3 \pi}{4} \frac{1}{\lambda}=\frac{3 \pi}{4} \sqrt{\frac{2 a_{0}}{5 M G}}=\frac{3 \pi}{4} \sqrt{\frac{4}{5} \frac{a_{0}}{a_{L}} R_{S} L} ; a_{L}=\frac{c^{2}}{L} .
$$

where $R_{S}=2 M G / c^{2}$ is the Schwarzschild radius and $L=c t_{H}$ is the Hubble scale $\left(t_{H}=13.8\right.$ Gyear). Because from calculation $a_{L} \approx 6.9 \times 10^{-10} \mathrm{~m} / \mathrm{s}^{2}$ and because $a_{0} \approx 1.25 \times 10^{-10} \mathrm{~m} / \mathrm{s}^{2}$ from MOND's assessment to most galaxies, if not all, we have from (43) for the Milky Way, where $R_{S} \approx 0.2$ lightyear,

$R_{M} \approx 458000$ lightyear.

This is well beyond the radius of the Milky Way, which amounts to $180.000-200.000$ lightyear. The coincidence range between MOND and this theory (up to $r \lambda=3 \pi / 4$ ) is well 
within the spatial validity range $r \lambda<6$ due to the weak field limit constraint and the linearization approximation. As noted before, apart from this upper limit for the range of validity, there is a lower limit as well. This has to do with the weak field limit constraint that we have imposed to derive a single parameter wave equation from Einstein's Field Equation. The value of this lower limit has been derived in the Appendix as

$R_{L} \approx\left(2 R_{S} \sqrt{\frac{a_{L}}{a_{0}}} \sqrt{\frac{5 R_{S} L}{2}}\right)^{1 / 2} \rightarrow R_{L} \approx\left(\frac{8}{3 \pi} R_{S} R_{M}\right)^{1 / 2}$

For the Milky Way ( $R_{S} \approx 0.2$ lightyear; $R_{M} \approx 458000$ lightyear) this lower limit amounts to

$R_{L} \approx 279$ lightyear.

Considering that our solar system is at about 26.000 lightyear from the center, it will be clear that the modified Newtonian gravitation law (35) holds for the Milky Way. Because many other galaxies are similar to the Milky Way, it is quite probable that this new theory solves the anomaly problem of the stellar rotation problem of most, if not all, galaxies.

From this result it may be concluded that that Milgrom's acceleration constant and Einstein's $\Lambda$ are closely related indeed. From (42) and (28), we have

$\lambda^{2}=2 \Lambda=\frac{2 a_{0}}{5 M G}$.

It also means that Einstein's $\Lambda$ is not a constant of nature, but instead, like noted before, a covariant integration constant that, while being independent of time-space coordinates, may be dependent on attributes of any cosmological system that is subject to Einstein's Field Equation. If the system is a spherical one, such as solar systems or galaxies, the value of Einstein's $\Lambda$ depends on the baryonic mass content of the system under consideration.

\section{The gravitational dipole density (2)}

Relationship (47) has been derived from the view that the vacuum is not an empty space, but instead a space filled with an energetic fluidum. As discussed earlier in this text, the molecules of the fluidum are vacuum particles in a state of Heisenberg unrest. These show an elementary dipole moment with a magnitude given by (12). The value of their actual mass is irrelevant. Up to recently, elementary fermions with a polarisable in a scalar potential field would have been just fictional particles, but Roza has shown that such particles may exist as third type Dirac particles [18]. Where such a dipole is imaginary for electron-type Dirac particles, and non-existing for Majorana-types, the third type shows such a particular property. And this property can be invoked for deriving a density expression for the vacuum particles. To do so, let us rewrite (29) as,

$\nabla^{2} \Phi+\lambda^{2} \Phi=-\frac{4 \pi G M}{c^{2}} \delta^{3}(r)$ 
and subsequently into,

$\nabla^{2} \Phi=-4 \pi G \rho(r)$, in which

$\rho(r)=\frac{M}{c^{2}} \delta^{3}(r)+\rho_{D}(r) ; \rho_{D}(r)=\frac{\lambda^{2}}{4 \pi G} \Phi(r)$.

In Debije's theory of electric dipoles [14,36,41],

$\rho_{D}(r)=-\nabla \cdot \mathbf{P}_{\mathbf{g}} \cdot$

The vector $\mathbf{P}_{\mathbf{g}}$ is the dipole density. From (50),

$\rho_{D}=\frac{1}{r^{2}} \frac{\mathrm{d}}{\mathrm{d} r}\left\{r^{2} P_{g}(r)\right\}$

Assuming that in the static condition the space fluid is eventually fully polarized by the field of the pointlike source, $P_{g}(r)$ is a constant $P_{g 0}$. Hence, from (51),

$\rho_{D}(r)=2 \frac{P_{g 0}}{r}$

Taking into account that to first order,

$\Phi(r)=\frac{M G}{r}$

we have from (52) and (53),

$\rho_{D}(r)=\frac{2 P_{g 0}}{M G} \Phi(r)$

Hence, from (47), (49) and (52-54),

$P_{g 0}=\frac{a_{0}}{20 \pi G}$

The volume density $N / \mathrm{m}^{3}$ of the darks is of course the same as the dipole density (55). Hence,

$N / \mathrm{m}^{3}=\frac{a_{0}}{20 \pi G}$ 


\section{A cosmological model of the universe}

So far, we have considered spherical cosmological systems under influence of a central gravitational force, such as applies to solar systems and galaxies. But what about the universe in terms of the relationships developed so far in this text? For any observer in the cosmos, the universe is a sphere with distributed matter. Let us model the universe as a sphere in the cosmos with radius $L$ and distributed gravitational energy. We have discussed before that vacuum is fluidal space with virtual sources $T_{\mu \mu}=-p \Lambda$, in which $p=c^{4} / 8 \pi G$. Denoting the gravitational background energy density as $\rho$, we have

$\rho c^{2}=\frac{c^{4}}{8 \pi G} \Lambda$

We have concluded before that $\Lambda$ is related with some baryonic mass $M_{B}$, such that

$$
\Lambda=\frac{a_{0}}{5 M_{B} G}
$$

The distributed energy is a gradually developed mixture of the energy from fluidal matter as meant by (57) and the energy from baryonic matter $M_{B}$ as meant by (58). From these expressions it can be concluded that the total gravitation energy $M_{G} c^{2}$ in a sphere with radius $L$ can be expressed as

$$
M_{G} c^{2}=\int_{0}^{L} \frac{a_{0}}{5 M_{B} G} \frac{c^{4}}{8 \pi G} 4 \pi r^{2} d r .
$$

Let $\Delta M_{G}$ the difference between the gravitational matter in a sphere $L+\Delta L$ and the gravitational matter in a sphere $L$. It follows readily that

$$
c^{2} \Delta M_{G}=\frac{a_{0}}{10 M_{B} G^{2}} c^{4} L^{2} \Delta L=\frac{a_{0}}{10 \Omega_{B} M_{G} G^{2}} c^{4} L^{2} \Delta L
$$

in which the baryonic matter is expressed as a dimensionless fraction $\Omega_{B}$ of the gravitational matter,

$$
M_{B}=\Omega_{B} M_{G} .
$$

Eq. (60) can now be integrated as

$$
\frac{1}{2} M_{G}^{2} c^{2}=\frac{a_{0}}{10 \Omega_{B} G^{2}} c^{4} \frac{L^{3}}{3} .
$$

Hence, the gravitational energy density $\rho c^{2}$ in the sphere with radius $L$ is given by 
$\rho c^{2}=\sqrt{\frac{a_{0}}{5 \Omega_{B} G^{2}} c^{6} \frac{L^{3}}{3}} \cdot \frac{3}{4 \pi L^{3}}=\frac{3}{4 \pi} \sqrt{\frac{a_{0}}{15 \Omega_{B} G^{2}} \frac{c^{6}}{L^{3}}}$.

Because the visible universe is a sphere from which light cannot escape, its radius equals the Schwarzschild radius,

$R_{S}=\frac{2 M_{U} G}{c^{2}} \rightarrow L=\frac{2 G}{2 c^{2}} \frac{4}{3} \pi L^{3} \rho ;$

in which $M_{U}$ is the total gravitational mass of the universe and in which $\rho$ is the overall matter density of the universe. Hence, from (63) and (64),

$\frac{3}{4 \pi} \sqrt{\frac{a_{0}}{15 \Omega_{B} G^{2}} \frac{c^{6}}{L^{3}}}=\frac{6 c^{4}}{2 G} \frac{1}{8 \pi L^{2}} \rightarrow \sqrt{\frac{a_{0} a_{L}^{3}}{15 \Omega_{B}}}=\frac{a_{L}^{2}}{2} \rightarrow a_{0}=\frac{15}{4} \Omega_{B} a_{L} ; a_{L}=\frac{c^{2}}{L}$.

Note that the relevancy of Milgrom's acceleration constant has now has been raised from the galaxy level up to the level of the visible universe. It is the same expression (17), which was found from an entropy point of view.

The baryonic energy density is just one of the three components of the gravitational energy. What about the other two components?

Let us inspect Einstein's Field Equation (18) once more,

$G_{\mu \nu}+\Lambda g_{\mu \nu}=\frac{8 \pi G}{c^{4}} T_{\mu \nu} ; G_{\mu \nu}=R_{\mu \nu}-\frac{1}{2} R g_{\mu \nu}$,

and let the metric of the spherically modelled visible universe be the well known FLRW metric [13], defined by the line element,

$$
\mathrm{d} s^{2}=\mathrm{d} q_{0}^{2}+a^{2}(t)\left(\frac{\mathrm{d} r^{2}}{1-k r^{2}}+r^{2} \sin ^{2} \vartheta \mathrm{d} \varphi^{2}+r^{2} \mathrm{~d} \vartheta^{2}\right)
$$

In which $q_{0}=\mathrm{i}$ ct is the normalized time coordinate $(\mathrm{i}=\sqrt{-1})$, and where $k$ is a measure for the curving of space-time. The scale factor $a(t)$ expresses the time-dependence of the size of the universe. The ratio

$\frac{\dot{a}}{a}=H(t)$

is known as the Hubble factor. It is the main observable of the universe, because its numerical value can be established from red shift observations on cosmological objects ( $\dot{a} \equiv \mathrm{d} a / \mathrm{d} t)$. The awareness that the vacuum is not an empty space, but, instead a fluidal one fluidal space with virtual sources $T_{\mu \mu}=-p \Lambda$, where $p=c^{4} / 8 \pi G[17,29,30]$ - owing to the Hawking metric, $p$ is equal for all diagonal elements - , will modify the two Friedmann 
equations [19] that originally have been conceived for empty space. This can be summarized as follows. First, the term $\Lambda g_{\mu \nu}$ is moved to the right side of (66), such that it can be conceived as an additional contribution to the energy density,

$\rho(t) \rightarrow \rho(t)+\rho_{\Lambda} ; \quad \rho_{\Lambda}=\frac{\Lambda}{\kappa} ; \kappa=\frac{8 \pi G}{c^{2}}$.

$p(t) \rightarrow p(t)-\rho_{\Lambda} c^{2}=0-\rho_{\Lambda} c^{2}$.

The solution of (69) under constraint of the metric (67) are [19],

$$
\begin{aligned}
& \left(\frac{\dot{a}}{a}\right)^{2}+\frac{k c^{2}}{a^{2}}=\frac{8 \pi G}{3} \rho \\
& 2 \frac{\ddot{a}}{a}+\left(\frac{\dot{a}}{a}\right)^{2}+\frac{k c^{2}}{a^{2}}=-\frac{8 \pi G}{c^{2}} p .
\end{aligned}
$$

Under the constraint $k=0$ (flat universe), and taking into consideration $(69 \mathrm{a}, \mathrm{b})$, the first Friedmann equation evolves as,

$$
\left(\frac{\dot{a}}{a}\right)^{2}=\frac{8 \pi G}{3}\left(\rho+\rho_{\Lambda}\right) \rightarrow\left(\frac{\dot{a}}{a}\right)^{2}=\frac{8 \pi G}{3} \rho_{c} ; \quad \rho_{t}=\rho+\rho_{\Lambda} .
$$

The second Friedmann equation reads as,

$\frac{\ddot{a}}{a}=\frac{4}{3} \pi G\left(2 \rho_{\Lambda}-\rho\right) \rightarrow \frac{\ddot{a}}{a}=\frac{4}{3} \pi G\left(2 \rho_{\Lambda}+2 \rho-3 \rho\right)=\frac{8}{3} \pi G \rho_{t}\left(1-\frac{3}{2} \alpha\right) ; \alpha=\frac{\rho}{\rho_{t}}$

Differentiating the mass density $\rho_{t}$ in (71a) gives,

$$
\begin{aligned}
& \dot{\rho}_{t}=\frac{3}{8 \pi G} \frac{\mathrm{d}}{\mathrm{d} t}\left(\frac{\dot{a}}{a}\right)^{2}=\frac{3}{8 \pi G}\left[2 \frac{\dot{a}}{a}\left\{\frac{\ddot{a}}{a}-\left(\frac{\dot{a}}{a}\right)^{2}\right\}\right]= \\
& 2 \frac{3}{8 \pi G} \frac{\dot{a}}{a}\left\{\frac{8}{3} \pi G\left(1-\frac{3}{2} \alpha\right)-\frac{8 \pi G}{3}\right\} \rho_{t}=2 \frac{\dot{a}}{a}\left\{\left(1-\frac{3}{2} \alpha\right)-1\right\} \rho_{t}=-3 \alpha \frac{\dot{a}}{a} \rho_{t} \rightarrow \\
& \dot{\rho}_{t}=-3 \alpha \frac{\dot{a}}{a} \rho_{t} \rightarrow \frac{1}{\rho_{t}} \frac{\mathrm{d} \rho_{t}}{\mathrm{~d} t}=-3 \frac{\rho}{\rho_{t}} \frac{1}{a} \frac{\mathrm{d} a}{\mathrm{~d} t} \rightarrow \frac{\mathrm{d} \rho_{t}}{\mathrm{~d} t}=-3 \rho \frac{1}{a} \frac{\mathrm{d} a}{\mathrm{~d} t} \rightarrow \\
& \frac{\mathrm{d} \rho}{\mathrm{d} t}+\frac{\mathrm{d} \rho_{\Lambda}}{\mathrm{d} t}=-3 \rho \frac{1}{a} \frac{\mathrm{d} a}{\mathrm{~d} t} .
\end{aligned}
$$

Because the background massive density $\rho_{\Lambda}$ is time-independent ( $\Lambda$ is independent of space-time coordinates), (72) is satisfied if,

$$
\frac{\mathrm{d} \rho}{\mathrm{d} t}=-3 \rho \frac{1}{a} \frac{\mathrm{d} a}{\mathrm{~d} t} \rightarrow \rho=\Omega_{m} H_{0} a^{-3} \quad \text { and } \quad \rho_{\Lambda}=H_{0} \Omega_{\Lambda}
$$


where $\Omega_{m}, \Omega_{\Lambda}$ and $H_{0}$ are constants. The quantity $H_{0}$ is the Hubble parameter $\dot{a} / a$ at $a(t)=1$. It is tempting to believe that $\Omega_{m}$ and $\Omega_{\Lambda}$ are, respectively, the relative amount of baryonic mass $\rho / \rho_{t}$ and the relative amount of background mass $\rho_{\Lambda} / \rho_{t}$ at $a(t)=1$. This, however, is not necessarily be true, because (without further constraints) the differential equation (69) is satisfied for any distribution between $\Omega_{m}$ and $\Omega_{\Lambda}$ as long as $\Omega_{m}+\Omega_{\Lambda}=1$.

Applying (73) on the first Friedmann equation (70a), results into,

$$
H(a)=\frac{\dot{a}}{a}=H_{0} \sqrt{\Omega_{m} a^{-3}+\Omega_{\Lambda}} .
$$

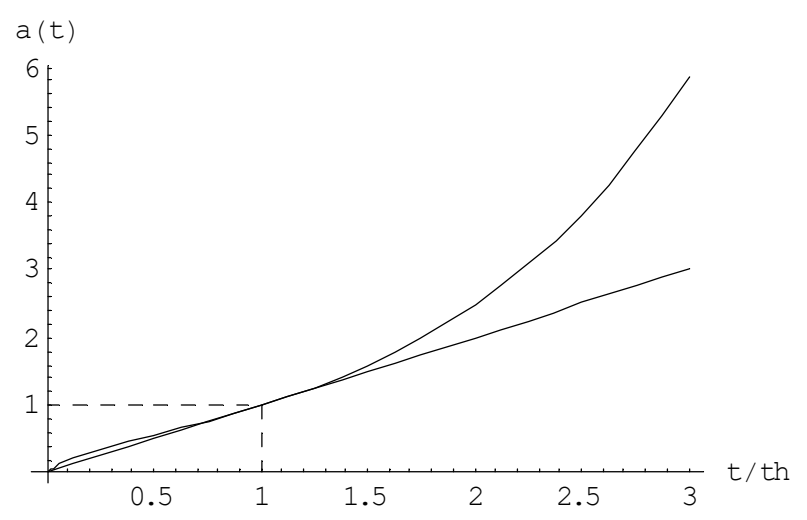

Fig.1: The scaling factor $a(t)$ as a function of cosmological time. The lower curve represents Hubble's law. The upper curve shows the curve of accelerated scaling due to Einstein's Cosmological Constant.

This equation represents the Lamda-CDM model in its most simple format (actually, more terms are heuristically added under the square root operator to model empirical evidence from certain cosmological phenomena). Eq. (74) can be analytically solved as $[42,43]$,

$$
a(t)=\left(\frac{\Omega_{m}}{\Omega_{\Lambda}}\right)^{1 / 3} \sinh ^{2 / 3}\left(3 \sqrt{\Omega_{\Lambda}} t / 2 t_{H}\right) ; t_{H}=H_{0}^{-1} .
$$

At present time $t=t_{P}$, the scale factor equals unity $(a=1)$ and the Hubble parameter is the observable $H_{0}$. Equating present time $t_{P}$ with Hubble time $t_{H}$ is justified if $a(t)$ would have shown a linear increase over time up to now, under a constant rate of say $c_{0}$, because in that case $a(t)=c_{0} t$ and $\dot{a}(t)=c_{0}$. This is Hubble's empirical law. Equating $t_{P}=t_{H}$ in (73) as an axiomatic assumption, indeed results in a behavior of the scale curve that, up to present time $t \leq t_{H}$, is pretty close to Hubble's empirical law. Hence, from (74),

$a\left(t_{H}\right)=1 ; H(a) \mid t_{H}=H_{0} \rightarrow \Omega_{m}+\Omega_{\Lambda}=1$.

Hence, from (75) and (74), 
$\left(\frac{1-\Omega_{\Lambda}}{\Omega_{\Lambda}}\right)^{1 / 3} \sinh ^{2 / 3}\left(3 \sqrt{\Omega_{\Lambda}} / 2\right)=1 \rightarrow \Omega_{\Lambda}=0.737 ; \Omega_{m}=0.263$

These values are only slightly different from those in the six-parameter Lamda-CDM model (where $\Omega_{m}=0.259$ ). The difference is due to the simplicity of the format (74), in which only matter and dark energy is included. Figure 1 demonstrates the viability of the axiomatic assumption to equate present time with Hubble time.

Summarizing:

From this paragraph:

The time behaviour of the scaling factor of the universe is a solution of Einstein's Field Equation under the FRLW-metric,

$$
a(t)=\left(\frac{\Omega_{m}}{\Omega_{\Lambda}}\right)^{1 / 3} \sinh ^{2 / 3}\left(3 \sqrt{\Omega_{\Lambda}} t / 2 t_{H}\right) ; t_{H}=H_{0}^{-1} .
$$

As a consequence of $a\left(t_{H}\right)=1$, the relative values for matter density and dark energy are established as,

$$
\Omega_{m}=0.263 \text { and } \Omega_{\Lambda}=0.737 \text {. }
$$

From the preceding paragraphs:

The relationship between Milgrom's acceleration parameter and the ratio $\Omega_{B}$ of baryonic matter over gravitational matter is established as,

$$
a_{0}=\frac{15}{4} \Omega_{B} a_{L}
$$

Accepting the life time of the universe $t_{H}=13.8$ Gyear and $a_{0}=1.256 \times 10^{-10} \mathrm{~m} / \mathrm{s}^{2}$ as primary independent quantities, we get $\Omega_{B}=0.0486$. This makes the dark matter content $\Omega_{D}=\Omega_{m}-\Omega_{B}=0.263-0.0486=0.214$.

\section{Discussion}

While under adoption of Hubble's law and the FLRW-metric the relationship between matter $\left(\Omega_{m}\right)$ and dark energy $\left(\Omega_{\Lambda}\right)$, as expressed by (76-77) is a straightforward consequence of Einstein's Field Equation, such as established in the Lambda-CDM ( $\mathrm{CDM}$ ) model of the Planck collaboration group [6], the relationship between dark matter $\left(\Omega_{D}\right)$ and baryonic matter $\left(\Omega_{B}\right)$ is in $\Lambda$ CDM empirically assessed. In this article, I have given it a theoretical basis. A crucial element in this, is the interpretation of Einstein's $\Lambda$ in his Field Equation. Usually, this quantity is considered as a constant of nature. In the context of $\Lambda C D M$ its value is established from (67) as a relationship between $\Lambda$ and gravitational matter, such that 
$\rho_{\Lambda} c^{2}=\Omega_{\Lambda} \rho_{c} c^{2}=\frac{c^{4}}{8 \pi G} \Lambda$

The critical mass density $\rho_{c}$ can be expressed in terms of Hubble time $t_{H}$ from the consideration that the universe is a bubble from which light cannot escape. Hence as a black hole with radius $R_{S}=c t_{H}$, such that

$R_{S}=c t_{H}=\frac{2 M_{c} G}{c^{2}} \rightarrow c t_{H}=\frac{2 G}{2 c^{2}} \frac{4}{3} \pi\left(c t_{H}\right)^{3} \rho_{c} \rightarrow \rho_{c} c^{2}=\frac{3}{8 \pi G} c^{2}\left(\frac{c}{t_{H}}\right)^{2}$.

From (81) and (82),

$$
\Lambda=\Omega_{\Lambda} \frac{3 a_{L}^{2}}{c^{4}} ; a_{L}=\frac{c}{t_{H}} . \quad\left(\approx 1.1 \times 10^{-52} \mathrm{~m}^{-2}\right)
$$

This expression assesses a numerical value of Einstein's $\Lambda$ at the level of the universe. At this level it makes sense to indicate this value as the Cosmological Constant. However, I have demonstrated in this article that in the application of Einstein's Field Equation at the level of solar systems and galaxies Einstein's $\Lambda$, while being a constant indeed in terms of independence of time-space coordinates, depends on the baryonic matter content $M_{B}$ of the system under consideration, such that

$$
\Lambda=\frac{1}{5} \frac{a_{0}}{M_{B} G}
$$

The dependence on the baryonic matter content is obviously present at the level of the universe as well, although it remained hidden as part of the critical mass content in the considerations (81-83). It wouldn't be correct to analyze the universe as a simple spherical system with a central gravitational force, like has been done in this article for galaxies. The universe is a distributed assembly of such spherical subsystems. It is for that reason that the critical mass of the universe, has been related in (59) to distributed baryonic kernels as fractions of gravitational matter. This allows not only to calculate the constant $a_{0}$ in a numerical value but also to establish it as a true cosmological invariant as a second gravitational constant next to the Newtonian $G$. It will be clear that calculation of Einstein's $\Lambda$ on the level of the universe from (84) as if the universe would behave similar as a galaxy is incorrect and, if done, will deliver a result that slightly deviates from (83). Curiously, where Einstein's $\Lambda$ is not a true cosmological constant, Milgrom's acceleration constant is. It has to be noted, though, that the description of the universe in the few simple parameters $a_{0}, G$ and $t_{H}$ is a status quo in the sense that there is no guarantee that the numerical values of the first two of these are true invariants over cosmological time, nor that Hubble's law has been true back to the big bang. 


\section{Conclusion}

The visible universe is a space filled with energetic vacuum particles that inherit their energy from their Heisenberg uncertainty in spatial position. It are gravitational quantum particles that possess a polarisable dipole moment with an eigen value $\hbar / 2 c$. In free state, the particle density is $\mathrm{N} / \mathrm{m}^{3}=a_{0} / 20 \pi G$, in which $a_{0}$ is a true cosmological invariant, with a numerical value equal to Milgrom's empirical acceleration constant for dark matter. If all these dipole moments were randomly orientated, the universe would show a perfect symmetry. The major part of these dipole moments $(\approx 74 \%)$ is randomly oriented indeed, because these don't feel a polarisable influence from a baryonic cluster. This part composes the dark energy of the universe. A significant part still $(\approx 21 \%)$ of the polarisable dipole moments are polarized around baryonic clusters. This part, with its frozen symmetries, composes the dark matter, which enhances the gravitational strength of the baryonic clusters. The baryonic clusters are densely packed energetic particles that composes the observable baryonic matter $\left(\Omega_{B} \approx 5 \%\right)$ of the universe. This amount is related with Milgrom's constant as $\Omega_{B}=4 t_{H} a_{0} / 15 c$.

\section{APPENDIX: THE GRAVITATIONAL WAVE EQUATION}

The main reason of including the appendix is to show the validity range for the weak field limited modification of Newton's gravitation law, due to Einstein's gauge constant $\Lambda$. To do so properly, the derivation requires a short summary of common textbook stuff without $\Lambda$, before extending it to meet the objective. This objective implies that we have to solve Einstein's Field Equation for a spherically symmetric space-time metric that is given by the line element (19),

$$
\mathrm{d} s^{2}=g_{t t}(r, t) \mathrm{d} q_{0}^{2}+g_{r r}(r, t) \mathrm{d} r^{2}+r^{2} \sin ^{2} \vartheta \mathrm{d} \varphi^{2}+r^{2} \mathrm{~d} \vartheta^{2}
$$

where $q_{0}=\mathrm{i} c t$.

Note: The space-time (ict, $r, \vartheta, \varphi$ ) is described on the basis of the "Hawking" metric $(+,+,+,+)$. Once more, I would like to emphasize its merit that, by handling time as an imaginary quantity instead of a real one, the ugly minus sign in the metric $(-,+,+,+)$ disappears owing to the obtained full symmetry between the temporal domain and the spatial one.

The components $g_{\mu \mu}$ compose the metric tensor $g_{\mu \nu}$, which determine the Ricci tensor $R_{\mu \nu}$ and the Ricci scalar $R$. These quantities play a decisive role in Einstein's Field Equation, which reads as

$G_{\mu \nu}+\Lambda g_{\mu \nu}=\frac{8 \pi G}{c^{4}} T_{\mu \nu} \quad$ with $G_{\mu \nu}=R_{\mu \nu}-\frac{1}{2} R g_{\mu \nu}$ 
In a space without massive sources, the Einstein Field Equation under this symmetric spherical isotropy, reduces to a simple set of equations for the elements $R_{\mu \mu}$ of the Ricci tensor,

$$
\begin{array}{ll}
R_{t t}-\frac{1}{2} R g_{t t}+\Lambda g_{t t}=0 ; & R_{r r}-\frac{1}{2} R g_{r r}+\Lambda g_{r r}=0 ; \\
R_{\vartheta \vartheta}-\frac{1}{2} R g_{\vartheta \vartheta}+\Lambda g_{\vartheta \vartheta}=0 ; & R_{\varphi \varphi}-\frac{1}{2} R g_{\varphi \varphi}+\Lambda g_{\varphi \varphi}=0 .
\end{array}
$$

Let us proceed by considering the Ricci scalar. It is defined generically as

$$
R=\sum_{\mu=0}^{3} \sum_{v=0}^{3} g^{\mu v} R_{\mu v} .
$$

In spherical symmetry the matrices contain diagonal elements only, so that $(A-4)$ reduces to

$$
R=\sum_{\mu=0}^{3} g^{\mu \mu} R_{\mu \mu}
$$

This result can be applied to (A-3). Multiplying the first one with $g^{00}\left(=g^{t t}\right)$, the second one with $g^{11}$, etc., and subsequent addition results of the terms $\mu=1,2,3$ gives,

$$
\begin{aligned}
& \sum_{\mu=1}^{3} g^{\mu \mu} R_{\mu \mu}-\frac{3}{2} R+3 \Lambda=-g^{t t} R_{t t}+\sum_{\mu=0}^{3} g^{\mu \mu} R_{\mu \mu}-\frac{3}{2} R+3 \Lambda=-g^{t t} R_{t t}-\frac{1}{2} R+3 \Lambda=0 \\
& \text { hence } g^{t t} R_{t t}=-\frac{1}{2} R+3 \Lambda
\end{aligned}
$$

Repeating this recipe for $g_{\mu \mu}\left(=1 / g^{\mu \mu}\right)$, we have for reasons of symmetry

$$
g^{\mu \mu} R_{\mu \mu}=-\frac{1}{2} R+3 \Lambda
$$

Note that the subscripts and superscripts $00,11,22$, and 33 are, respectively, identical to $t t, r r, \vartheta \vartheta$ and $\varphi \varphi$. Applying this result to Einstein's equation set gives,

$$
2 g^{\mu \mu} R_{\mu \mu}-2 \Lambda=\frac{8 \pi G T_{\mu \mu} g^{\mu \mu}}{c^{4}}
$$

such that after multiplication by $g_{\mu \mu}$, we have

$$
2 R_{\mu \mu}-2 g_{\mu \mu} \Lambda=\frac{8 \pi G T_{\mu \mu}}{c^{4}}
$$


Let us first proceed under the conditions of the absence of massive sources $\left(T_{\mu \mu}=0\right)$ and let us consider the Ricci tensor components $R_{t t}$ and $R_{r r}$ under use of the results shown in Table A-1, that can be found in basic textbooks [31]. Note: $g^{\prime}$ and $g^{\prime \prime}$ means differentiation, respectively double differentiation of $g$ into $r ; \dot{g}$ and $\ddot{g}$ means differentiation, respectively double differentiation of $g$ into $t$. Multiplying (A-3a) by $1 / g_{t t}$ and (A-3b) by $1 / g_{r r}$ gives,

$\frac{R_{t t}}{g_{t t}}-\Lambda=0$ and $\frac{R_{r r}}{g_{r r}}-\Lambda=0$

which, under the assumption of a zero Cosmological Constant $(\Lambda=0)$, after subtraction and under use of the expressions in Table A-1 results into.

$-\frac{1}{r} \frac{1}{g_{r r}}\left(\frac{g_{r r}^{\prime}}{g_{r r}}+\frac{g_{t t}^{\prime}}{g_{t t}}\right)=0$

Hence

$\frac{g_{r r}^{\prime}}{g_{r r}}+\frac{g_{t t}^{\prime}}{g_{t t}}=0$

which can be integrated to (the Schwarzschild condition),

$g_{r r} g_{t t}=1$.

This, in turn, gives

$\frac{\dot{g}_{r r}}{g_{r r}}+\frac{\dot{g}_{t t}}{g_{t t}}=0$

Using (A-13), (A-15) and the Table A-1 values on $R_{t t}$ gives

$$
R_{t t}=\frac{1}{g_{r r}}\left(-\frac{1}{2} g_{t t}^{\prime \prime}-\frac{1}{r} g_{t t}^{\prime}-\frac{\ddot{g}_{r r}}{2 c^{2}}\right)=\frac{1}{g_{r r}}\left(-\frac{1}{2 r} \frac{\partial^{2}\left(r g_{t t}\right)}{\partial r^{2}}+\frac{1}{2 c^{2}} \frac{\partial^{2} g_{t t}}{\partial t^{2}}\right)
$$

Hence, from (A-10) and (A-16),

$$
\frac{2}{g_{r r}}\left(-\frac{1}{2 r} \frac{\partial^{2}\left(r g_{t t}\right)}{\partial r^{2}}+\frac{1}{2 c^{2}} \frac{\partial^{2} g_{t t}}{\partial t^{2}}\right)=\frac{8 \pi G T_{t t}}{c^{4}}
$$

or, equivalently,

$$
-\frac{1}{r} \frac{\partial^{2}\left(r g_{t t}\right)}{\partial r^{2}}+\frac{1}{c^{2}} \frac{\partial^{2} g_{t t}}{\partial t^{2}}=\frac{8 \pi G T_{t t}}{c^{4}} g_{r r}
$$


Applying well-known conditions,

$\Lambda=0 \quad$ (already assumed) (no cosmological constant)

$g_{t t}(r, t)=1+h_{\varphi}(r, t)$, where $\left|h_{\varphi}(r, t)\right|<<1 \quad$ (the weak field limit)

$T_{t t}=M c^{2} \delta^{3}(r), \quad$ (pointlike massive source) $\quad$ (A-19a, b,c)

yields the proper wave equation

$\frac{\partial^{2}\left(r h_{\varphi}\right)}{\partial r^{2}}-\frac{1}{c^{2}} \frac{\partial^{2} r h_{\varphi}}{\partial t^{2}}=-\frac{8 \pi G M}{c^{2}} r \delta^{3}(r) U(t)$,

where $U(t)$ is Heaviside's step function. In the static regime, the equation results into

$$
\frac{1}{r} \frac{\partial^{2}\left(r h_{\varphi}\right)}{\partial r^{2}}=-\frac{8 \pi G M}{c^{2}} \delta^{3}(r) .
$$

This is similar to Poisson's equation,

$$
\nabla^{2} \Phi=\frac{1}{r} \frac{\partial^{2}(r \Phi)}{\partial r^{2}}=-4 \pi G \rho=-4 \pi G M \delta^{3}(r)
$$

the solution of which is the Newtonian potential,

$$
\Phi=-\frac{G M}{r}\left[\mathrm{~m}^{2} \mathrm{~s}^{-2}\right]
$$

Comparing (A-20) with (A-22) gives the equivalence

$$
h_{\varphi}=\frac{2 \Phi}{c^{2}}
$$

So far, this is just textbook stuff, such as can be found, for example, in [31]. It is needed as a basis for deriving the conditions under which the modification of the $\Lambda=0$ wave equation (A-20) toward the $\Lambda \neq 0$ one shown in (28) is justified. Let us first consider the case $\Lambda \neq 0$ under absence of a massive source. Obviously, (A-10) is only satisfied if the influence of the cosmological constant is counter balanced by the hypothetical source

$$
T_{t t}=-p \Lambda, \text { where } p=\frac{c^{4}}{8 \pi G}
$$

Because all four members of the Einstein set $(A-10)$ have to be satisfied, we have, under consideration of $(A-10)$ and Table $A 1$, 
$T_{\mu \mu}=-p g_{\mu \mu} \Lambda$ and $g_{\mu \mu}=\left(1,1, r^{2} \sin ^{2} \vartheta, r^{2}\right)$

This particular stress-energy tensor with equal diagonal elements corresponds with the one for a perfect fluid in thermodynamic equilibrium. So, where empty space corresponds with virtual sources $T_{\mu \mu}=0$, the fluidal space corresponds with virtual sources $T_{\mu \mu}=-p \Lambda$, with $g_{\mu \mu}=\left(1,1, r^{2} \sin ^{2} \vartheta, r^{2}\right)$. Insertion of a massive pointlike source in this fluid and modifying (A-17) by adding the virtual sources, after redefining the weak limit condition as,

$g_{t t}(r, t)=1+h_{t \varphi}(r, t) ; g_{t r}(r, t)=1+h_{r \varphi}(r, t) ;\left|h_{t \varphi}\right|,\left|h_{r \varphi}\right|<<1$,

gives, for the static parts,

$$
\begin{aligned}
& \left.\frac{2}{g_{r r}}\left\{-\frac{1}{2} h_{t \varphi}^{\prime \prime}+\frac{h_{t \varphi}^{\prime}}{4}\left(h_{r \varphi}^{\prime}+h_{t \varphi}^{\prime}\right)-\frac{1}{r} h_{t \varphi}^{\prime}\right)\right\}-2 \Lambda g_{t t}=\frac{8 \pi G T_{t t}}{c^{4}}-2 \Lambda \rightarrow \\
& -h_{t \varphi}^{\prime \prime}-2 \Lambda h_{t \varphi}+\frac{h_{t \varphi}^{\prime}}{2}\left(h_{r \varphi}^{\prime}+h_{t \varphi}^{\prime}\right)-\frac{2}{r} h_{t \varphi}^{\prime}=\frac{8 \pi G T_{t t}}{c^{4}} g_{r r}
\end{aligned}
$$

and, secondly,

$-h_{t \varphi}^{\prime \prime}-2 \Lambda h_{r \varphi}+\frac{h_{t \varphi}^{\prime}}{2}\left(h_{r \varphi}^{\prime}+h_{t \varphi}^{\prime}\right)+\frac{2}{r} h_{r \varphi}^{\prime}=0$

As long as $\Lambda=0$, and assuming a pointlike source embodied in $T_{t t}$, the Schwarzschild condition shows up. This is obvious by subtracting the latter equation from the former one, thereby allowing exclusion of a singularity at $r=0$. It reveals that, under this condition, the homogeneous formats of the two equations are identical. However, because this is no longer true for $\Lambda \neq 0$, we have to cope with two equations. These two equations are non-linear. However, because $h_{t \varphi}$ and $h_{r \varphi}$ are small in the weak field limit, the two equations can be linearized under the condition that the last term in the left-hand part of these equations is dominant over their preceding terms. This assumption, to be checked later, allows to rewrite (A-26) for $r \neq 0$, as

$\left(r h_{t \varphi}\right)^{\prime \prime}+2 \Lambda\left(r h_{t \varphi}\right)=0$

A simple format for the second equation is obtained after subtraction (A-27) from (A-26), resulting into,

$-2 h_{r \varphi}^{\prime}+2 \Lambda r h_{r \varphi}=2 h_{t \varphi}^{\prime}+2 \Lambda r h_{t \varphi}$.

Obviously, $h_{r \varphi}$ can be calculated as soon as $h_{t \varphi}$ is found as a solution of (A-28). Re-inserting the pointlike source, similarly as in the case $\Lambda=0$ and including the time derivatives, yield a wave equation as a generalization of (A-28). After rewriting, 
$h_{t \varphi}=\frac{2 \Phi}{c^{2}}$ and $\Lambda=\frac{\lambda^{2}}{2}$,

we have from (A-28) the inhomogeneous generalization,

$$
-\frac{\partial^{2}}{c^{2} \partial t^{2}}(r \Phi)+\frac{\partial^{2}}{\partial r^{2}}(r \Phi)+\lambda^{2}(r \Phi)=-r \frac{8 \pi G M}{c^{2}} \delta^{3}(r) U(t)
$$

If $\Lambda<0$, we have under static conditions, a similarity with Helmholtz' equation [54] with the screened Poisson's equation, the solution of which is Yukawa's potential,

$\Phi=\frac{G M}{r} \exp (-\lambda r)$

which reduces to Poisson's one for $\lambda \rightarrow 0$.

If $\Lambda>0$, we have under static conditions, a similarity with Helmholtz' equation with a characteristic solution,

$\Phi=\frac{G M}{r}\{\cos \lambda r+\sin \lambda r\}$

This solution reduces to Poisson's one for $\lambda \rightarrow 0$ as well.

This is the weak field limit solution of Einstein's Equation if one does not take the validity of Poisson's equation of gravity for granted, but adopts Helmholtz equation instead under an appropriate choice of the Cosmological Constant.

We are not done yet. There are two remaining issues. The first one is the justification of the linearization approximation by moving from (A-26) to (A-28). Moreover, we have to take into consideration that, although the derived gravitational potential field satisfies $(A-3 a)$ and $(A-$ $3 b)$, we are not sure that it satisfies $(A-3 c)$ and $(A-3 d)$ as well. It should do, to prevent violation of the metric (A-1). Assessment of it is the second thing to be done.

The remaining issues: (a) the linearization approximation

The linearity approximation $(A-26) \rightarrow(A-28)$ is justified as long as

$\left|\frac{2}{r} h_{t \varphi}^{\prime}\right|>>\left|\frac{h_{t \varphi}^{\prime}}{2}\left(h_{r \varphi}^{\prime}+h_{t \varphi}^{\prime}\right)\right| \rightarrow \frac{1}{r}>>\frac{1}{4}\left|h_{r \varphi}^{\prime}+h_{t \varphi}^{\prime}\right|$.

Under consideration of (A-29), it can be written as,

$\frac{1}{\Lambda r^{2}}>>\frac{1}{4}\left|h_{r \varphi}-h_{t \varphi}\right|$

This condition enforces calculation of $h_{r \varphi}$ from $h_{t \varphi}$. From (A-29), 


$$
h_{r \varphi}^{\prime}-\frac{\lambda^{2} r}{2} h_{r \varphi}=-\left(h_{t \varphi}^{\prime}+\frac{\lambda^{2} r}{2} h_{t \varphi}\right)
$$

This first order differential equation for $h_{r \varphi}$ can be readily solved, albeit that the resulting analytical expression from the generic solution

$$
\begin{aligned}
& h_{r \varphi}(\lambda r)=\frac{1}{p(\lambda r)} \int p(\lambda r) f(\lambda r) d(\lambda r), \text { in which } \\
& f(\lambda r)=\frac{\partial}{\partial(\lambda r)} h_{t \varphi}(\lambda r)+\frac{\lambda r}{2} h_{t \varphi}(\lambda r) ; p(\lambda r)=\exp \left\{\int-\frac{\lambda r}{2} d(\lambda r)\right\}=\exp \left(-\frac{\lambda^{2} r^{2}}{4}\right),
\end{aligned}
$$

is a rather complicated one.

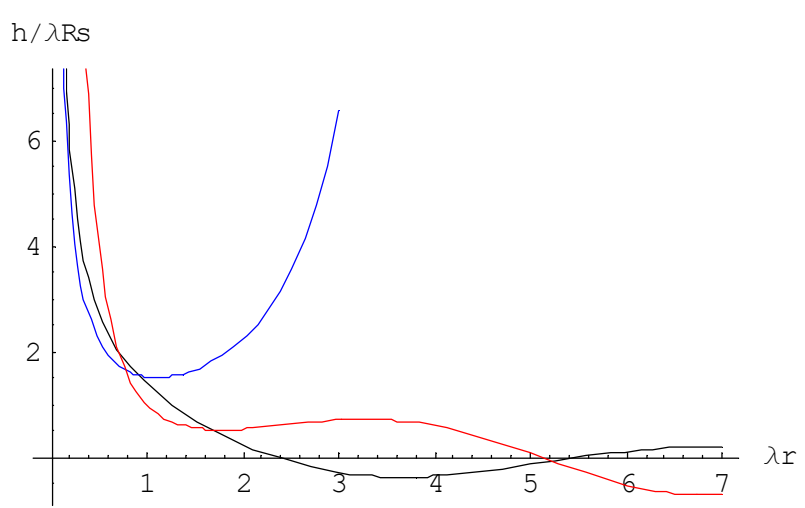

Fig. A-1: Relative values of the metric quantities $h_{t \varphi}$ (black) and $-h_{r \varphi}$ (blue) as a function of $\lambda r$. The red curve represents the function in the right-hand part of (A-36).

Figure A-1 illustrates the behavior of the calculated $-h_{r \varphi}$ as a function of $\lambda r$ compared with $h_{t \varphi}$. From (A-36), it is obvious that if $\lambda r \rightarrow 0, h_{r \varphi} \approx-h_{t \varphi}$. The vertical axis is normalized to a dimensionless quantity, by writing, under consideration of (A-30) and (A-33),

$h_{t \varphi}=\frac{2}{c^{2}} \frac{G M}{r}\{\cos \lambda r+\sin \lambda r\}=\frac{\lambda R_{S}}{\lambda r} \cos \lambda r+\sin \lambda r ; R_{S}=\frac{2 M G}{c^{2}}$.

Note that $R_{S}$ is the Schwarzschild radius of the cosmological system (with central force) under consideration. The black curve shows the normalized value of $h_{t \varphi}$. It is gradually decreasing by $r^{-1}$. The blue curve shows the normalized value of $h_{r \varphi}$ as calculated from the differential equation (A-36). This quantity tend to explode with increasing $\lambda r$. Nevertheless the functions on both sides of (A-36) remain the same and show the gradual finite behavior, shown by the red curve. The reason is due to sign differences between the left-hand part and the right-hand part of (A-35). Subtraction of two large quantities makes the result still small enough. Nevertheless, the exponential increase of $h_{r \varphi}$ may violate the linearization 
approximation. This requires proper investigation. Because eventually (for relatively large $\lambda r) h_{r \varphi} \gg h_{t \varphi}$, and considering that $2 \Lambda=\lambda^{2}$, we may reformulate (A-35) as,

$\frac{1}{\lambda r}>>\frac{\lambda}{8} R_{S}\left(\frac{h_{r \varphi}}{\lambda R_{S}}\right)$

Hence, the cross over value $\lambda r_{0}$ is determined as,

$$
\lambda r_{0}=\frac{8}{h_{r \varphi}\left(r_{0}\right)}=\frac{8}{\lambda R_{S} h_{r \varphi}\left(\lambda r_{0}\right)}
$$

where $h_{r \varphi}(\lambda r)$ is given by (A-37). From (A-39) it is obvious that as long as $h_{r \varphi}<<1$, the upper limit is far beyond $\lambda r=8$. However, because of the exponential growth of $h_{r \varphi} / \lambda R_{S}$ with $\lambda r$, the limit can be shifted near to this limit or even shifted below. This may spoil the weak field limit assumption. Hence, the actual validity range of the linearization heavily depends on the value of the product $\lambda R_{S}$. Once this product is known, the cross-over value of $\lambda r_{0}$ and the associated value of the metric component $h_{r \varphi}$ can be calculated from the known curve $h_{r \varphi} / \lambda R_{S}$ shown in fig. A-1. Because of its exponential growth, the upper limit for $\lambda r$ that justifies the linearization approximation, is below, but probably near, to the cross-over value.

The assessment of a meaningful quantitative value to the product $\lambda R_{S}$ is possible by invoking the value of Einstein's $\Lambda$ for cosmological systems with a central mass. As shown in the main text, this is obtained by the application of the theory to Milgrom's MOND, expressed by eq. (26). This expression relates Einstein's Cosmological Constant $\Lambda$ with Milgrom's acceleration constant $a_{0}$ as,

$2 \Lambda=\lambda^{2}=\frac{2 a_{0}}{5 M G}$

Hence,

$$
\lambda R_{S}=2 \sqrt{\frac{a_{0}}{a_{L}} \frac{R_{S}}{L}} ; a_{L}=\frac{c^{2}}{L},
$$

where $a_{L}$ is the gravitational acceleration constant at distance $L$ from the centre of the cosmological system under consideration. Choosing $L$ as the Hubble range $L=c t_{H}$ and defining $c^{2} / L=a_{L}$ as the acceleration at the verge of the Hubble range, and considering that Milgrom's acceleration constant amounts to $a_{0} \approx 1.25 \times 10^{-10} \mathrm{~m} / \mathrm{s}^{2}$, the ratio $a_{L} / a_{0}$ amounts to, $a_{L} / a_{0} \approx 6.9 / 1.25=5.52$. The Schwarzschild radius $R_{S}$ of a typical galaxy, like the Milky Way, is about 0.2 lightyear, while the Hubble time amounts to $t_{H} \approx 13.5$ Gyear. Hence, typically 
$\lambda R_{S} \approx 3.3 \times 10^{-6}$.

From (A-42), (A-40) and (A-37), it is found that the cross-over $\lambda r_{0}$ amounts to

$\lambda r_{0} \approx 7.25$.

The associated value of the metric component amounts to $h_{r \varphi}=1.01$. That violates the weak field approximation. However, at $\lambda r=6$, the metric component is drops to $h_{r \varphi}=0.017$. Hence, It is fair to say that, up to a normalized spatial distance near to $\lambda r_{0} \approx 6$, the derived gravitational wave equation (A-31) for galaxies akin to the Milky Way maintains its validity.

The remaining issues: (b) the other two equations

Before appearance of the massive source, we have for $(A-3 c)$,

$\left\{1+\frac{r}{2 g_{r r}}\left(\frac{g_{r r}^{\prime}}{g_{r r}}-\frac{g_{t t}^{\prime}}{g_{t t}}\right)-\frac{1}{g_{r r}}\right\}-g_{\vartheta \vartheta} \Lambda=$ background matter,

where $g_{\mu \mu}=\left(1,1, r^{2} \sin ^{2} \vartheta, r^{2}\right)$

After appearance of the massive source, we have

$1+\frac{r}{2 g_{r r}}\left(\frac{g_{r r}^{\prime}}{g_{r r}}-\frac{g_{t t}^{\prime}}{g_{t t}}\right)-\frac{1}{g_{r r}}-g_{\vartheta \vartheta} \Lambda=$ background matter plus source,

where $g_{\mu \mu} \neq\left(1,1, r^{2} \sin ^{2} \vartheta, r^{2}\right)$.

Due to the change of curving by the source, we have,

$1+\frac{r}{2 g_{r r}}\left(\frac{g_{r r}^{\prime}}{g_{r r}}-\frac{g_{t t}^{\prime}}{g_{t t}}\right)-\frac{1}{g_{r r}}-\left(g_{\vartheta \vartheta}-r^{2}\right) \Lambda=0$.

Under the constraint of the weak field limit, this equation can be rewritten as,

$$
\Lambda g_{\vartheta \vartheta}=\Lambda r^{2}-r h_{t \varphi}^{\prime}-h_{t \varphi}+\frac{r}{2}\left(h_{r \varphi}^{\prime}+h_{t \varphi}^{\prime}\right)+\left(h_{r \varphi}+h_{t \varphi}\right)=0
$$

As long as $\Lambda g_{\vartheta \vartheta}$ is close to $\Lambda r^{2}$, the metric (A-1) maintains it validity. This is true as long as

$$
\begin{aligned}
& r^{2}>>\frac{1}{\Lambda}\left(r h_{t \varphi}^{\prime}+h_{t \varphi}\right), \text { and } \\
& r^{2} \gg>\frac{1}{\Lambda}\left\{\frac{r}{2}\left(h_{r \varphi}^{\prime}+h_{t \varphi}^{\prime}\right)+\left(h_{r \varphi}+h_{t \varphi}\right)\right\} .
\end{aligned}
$$


The split into two conditions is made for ease of analysis. Under consideration of (A-29), (A48b) can be rewritten as,

$$
r^{2}>>\frac{1}{\Lambda}\left\{\left(1+\frac{\lambda^{2} r^{2}}{2}\right) h_{r \varphi}+\left(1-\frac{\lambda^{2} r^{2}}{2}\right) h_{t \varphi}\right\}
$$

Because with increasing $\lambda r$ the quantity $h_{r \varphi}$ is dominating over $h_{t \varphi},(\mathrm{A}-50)$ can be replaced by,

$$
r^{2}>>\frac{1}{\Lambda}\left(\frac{\lambda^{2} r^{2}}{2}\right) h_{r \varphi} \rightarrow r^{2}>>r^{2} h_{r \varphi} \rightarrow h_{r \varphi}<<1
$$

thereby concluding that the condition $(A-49 b)$ is covered by the weak field constraint.

Now we have established the upper spatial limit justified the linearization condition and concluded that condition ( $\mathrm{A}-48 \mathrm{~b}$ ) is covered by the weak field constraint, we are left with a single issue. That is condition (A-48a). Considering that,

$$
\begin{aligned}
& h_{t \varphi}=\frac{2}{c^{2}} \frac{G M}{r}\{\cos \lambda r+\sin \lambda r\} ; h_{t \varphi}^{\prime}=-\frac{R_{S}}{r^{2}}\{\cos \lambda r+\sin \lambda r\}+\frac{R_{S}}{r}(-\lambda \sin \lambda r+\lambda \cos \lambda r) \\
& h_{t \varphi}^{\prime}=-\frac{R_{S}}{r^{2}}\{(\lambda r-1) \cos \lambda r-(1+\lambda r) \sin \lambda r\},
\end{aligned}
$$

we have for (A-49a),

$$
\begin{aligned}
& r^{2}>>\frac{R_{S}}{\Lambda}(-\lambda \sin \lambda r+\lambda \cos \lambda r) \rightarrow \\
& r^{2}>>\frac{2 R_{S}}{\lambda}(-\sin \lambda r+\cos \lambda r) \approx r^{2}+\frac{2\left|R_{S}\right|}{\lambda}=r^{2}+\frac{2\left|R_{S}\right|}{\sqrt{2 a_{0}}} c \sqrt{5 M G / c^{2}}= \\
& r^{2}>>\frac{2\left|R_{S}\right|}{\sqrt{2 a_{0}}} c \sqrt{\frac{5 R_{S}}{2}}=\frac{2\left|R_{S}\right|}{\sqrt{2 a_{0}}} \sqrt{\frac{c^{2}}{L}} \sqrt{\frac{5 R_{S}}{2}} \sqrt{L}=2\left|R_{S}\right| \sqrt{\frac{a_{L}}{a_{0}}} \sqrt{\frac{5 R_{S} L}{2}} .
\end{aligned}
$$

Hence,

$r>>R_{G}$, where $R_{G}=\left(2\left|R_{S}\right| \sqrt{\frac{a_{L}}{a_{0}}} \sqrt{\frac{5 R_{S} L}{2}}\right)^{1 / 2}$.

As already noted, the Schwarzschild radius $R_{S}$ of a typical galaxy, like the Milky Way, is about 0.2 lightyear. For such a galaxy, the range $R_{G}$ calculated from (A-54) appears being $R_{G} \approx 278$ lightyear. Considering that the radius of the Milky Way is estimated as $100.000-180.000$ lightyear and that our solar system is at about 26.000 lightyear from the center, it will be clear that the wave equation (A-31) holds for the major part of the galaxy, thereby solving the anomaly problem of the stellar rotation problem. 
Table A1: metric tensor and Ricci tensor

\begin{tabular}{|l|l|}
\hline metric tensor & Ricci tensor \\
\hline$g_{t t} \equiv g_{00}$ & $R_{t t}=-\frac{1}{2} \frac{g_{t t}^{\prime \prime}}{g_{r r}}-\frac{\ddot{g}_{r r}}{2 c^{2} g_{r r}}+\frac{g_{t t}^{\prime}}{4 g_{r r}}\left(\frac{g_{r r}^{\prime}}{g_{r r}}+\frac{g_{t t}^{\prime}}{g_{t t}}\right)-\frac{\dot{g}_{r r}}{4 c^{2} g_{r r}}\left(\frac{\dot{g}_{r r}}{g_{r r}}+\frac{\dot{g}_{t t}}{g_{t t}}\right)-\frac{1}{r} \frac{g_{t t}^{\prime}}{g_{r r}}$ \\
\hline$g_{r r} \equiv g_{11}$ & $R_{r r}=-\frac{1}{2} \frac{g_{t t}^{\prime \prime}}{g_{t t}}-\frac{\ddot{g}_{r r}}{2 c^{2} g_{t t}}+\frac{g_{t t}^{\prime}}{4 g_{t t}}\left(\frac{g_{r r}^{\prime}}{g_{r r}}+\frac{g_{t t}^{\prime}}{g_{t t}}\right)-\frac{\dot{g}_{r r}}{4 c^{2} g_{t t}}\left(\frac{\dot{g}_{r r}}{g_{r r}}+\frac{\dot{g}_{t t}}{g_{t t}}\right)+\frac{1}{r} \frac{g_{r r}^{\prime}}{g_{r r}}$ \\
\hline$g_{\vartheta \vartheta} \equiv g_{22}=r^{2}$ & $R_{\vartheta \vartheta}=1+\frac{r}{2 g_{r r}}\left(\frac{g_{r r}^{\prime}}{g_{r r}}-\frac{g_{t t}^{\prime}}{g_{t t}}\right)-\frac{1}{g_{r r}}$ \\
\hline$g_{\varphi \varphi} \equiv g_{33}=r^{2} \sin ^{2}(\vartheta)$ & $R_{\kappa \varphi}=\sin ^{2}(\vartheta) R_{\vartheta \vartheta}$ \\
\hline
\end{tabular}

\section{References}

[1] Shu-Kun Lin, J. Chem. Inf. Comput. Sci, 36, 367 (1996)

[2] E. Verlinde, JHEP 1104, 029 ; arXiv: 1001.0785v1 [hep-th] (2011)

[3] E. Verlinde, SciPost Phys. 2, 016 (2017); arXiv: 1611.02269v2 [hep-th] (2016)

[4] T. Jacobson, Phys. Rev. Lett., 75, no. 7, 1260 (1995)

[5] M. Milgrom, The Astrophysical Journal, 270, 365 (1983)

[6] Planck Collaboration, Astronomy and Astrophys., 594: A13 (2015)

[7] J.D. Bekenstein, Phys. Rev. D7, 2333 (1973)

[8] S.W. Hawking, Comm. Math. Phys. 43, 199 (1975)

[9] L. Susskind, Inside blackholes, lecture on youtube

[10] R.K. Pathria, Nature, 240 (5379, 298 (1972)

[11] L.I. Mandelshtam, I.E. Tamm, J. Phys (USSR) 9, 249 (1945)

[12] http://vergil.chemistry.gatech.edu/notes/ho/node2.html

[13] L. Blanchet, Class. Quantum Grav. 24, 3529 (2007)

[14] D. Hajdukovic, Astrophysics and Space Science, 334, vol.2, 215 (2011)

[15] A. Einstein, Preuss. Akad. Wiss, Berlin (Math. Phys.), 142 (1917)

[16] www.scholarpedia.org/article/Cosmological constant

[17] S. Carroll, W. Press and E. Turner, Ann. Rev. Astronomy and Astrophys, 30, 499 (1992)

[18] E. Roza, Found. on Phys. , 50 (8), 828 (2020)

[19] A. Friedmann, Zeitschrift fur Physik A, 10(1), 377 (1922)

[20] R. d'Inverno, Introducing Einstein's Relativity, Ch. 23, Oxford Press (1992)

[21] G. Gentile, B. Famae and W.G.J . de Blok, J. Astronomy and Astrophysics, 527, 76 (2011)

[22] A. Einstein, Relativity: The Special and General Theory, H. Holt and Company, New York (1916, translation 1920)

[23] S. Weinberg, Gravitation and Cosmology, John Wiley \& Sons, Inc., New York (1972)

[24] T.A. Moore, A General Relativity Workbook, University Science Books, (2013)

[25] A. Einstein, Ann. der Physik, 4 (55), 241 (1918)

[26] D. Perkins, Introduction to High Energy Physics, $4^{\text {th }}$ Ed., Cambridge Univ. Press, Cambridge UK (2000)

[27] F. Kottler, Ann. Physik 56,361,401 (1918)

[28] Li-Feng Sun et al., Modern Phys. Lett. A 28, 1350114 (2013)

[29] S. Carroll, W. Press and E.Turener, Ann. Rev. Astronomy and Astrophys. 30, 499 (1992)

[30] J. Sola, Journal of Physics, Conf. Series, 453, 012015 (2013) 
[31] B. Schutz, A First Course in General Relativity, $2^{\text {nd }}$ ed, Cambridge Univ. Press, New York (2009) [32] J.D. Norton, The Cosmological Woes of Newtonian Gravitation Theory, Einstein studies, 7. Birkauser, Boston, 217, ISBN 0817640606 (1999)

[33] A. Harvey, E.. Schucking, Am. Journ. of Physics, Vol. 68, Issue 8, 723 (2000).

[34]H. Yukawa, Proc. Phys. Math. Soc. Jpn 17, 48 (1935); H. Yukawa and S. Sakata, Proc. Phys. Math. Soc. Jpn., 19, 1084 (1937)

[35] A. Proca, J. Phys. Radium, 7, 346 (1936); Acad. Sci. Paris, C.R. 202, 1366 (1936)

[36]P. Debye and E. Huckel, Physik. Zetschrift, vol. 24, 9, 185 (1923)

[37] V. Rubin, N. Thonnard, W.K. Ford Jr., Astrophysical Journal, 238, 471 (1980)

[38] A.V. Minkevich, Acta Physica Polonica Series B, 38, 1 (2006)

[39] O. Luongo, H. Cuevedo, Found. of Phys. 48, 17, doi:10.1007/s10701-017-0125-0 (2018)

[40] V.A. Etkin, Journ. Appl. Phys. vol. 8, issue 6 , 43, doi:10.9790.4861-0806054349 (2016)

[41] C.A. Gonano, R.E. Zich, M. Mussetta, Progr. in Electromagn. Res. B, 64, 83 (2015)

[42] S. Wolfram, Mathematica, Addison Wesley, Redwood City A (1991)

[43] https://en.wikipedia.org/wiki/Lambda-CDM model (accessed July 25, 2020) 